\title{
Hybrid modeling of collaborative freight transportation planning using agent-based simulation, auction-based mechanisms, and optimization
}

\author{
Ki-Hwan Bae, Industrial Engineering, Colorado Christian University, USA \\ Navonil Mustafee, CSAM, University of Exeter Business School, UK \\ Sanja Lazarova-Molnar, University of Southern Denmark, Denmark \\ Long Zheng, University of Louisville, USA
}

The sharing economy is a peer-to-peer economic model characterized by people and organizations sharing resources. With the emergence of such economies, an increasing number of logistics providers seek to collaborate and derive benefit from the resultant economic efficiencies, sustainable operations, and network resilience. This study investigates the potential for collaborative planning enabled through a Physical Internetenabled logistics system in an urban area that acts as a freight transport hub with several ecommerce warehouses. Our collaborative freight transportation planning approach is realized through a three-layer structured hybrid model that includes agent-based simulation, auction mechanism, and optimization. A multi-agent model simulates a complex transportation network, an auction mechanism facilitates allocating transport services to freight requests, and a simulation optimization technique is used to analyze strategic transportation planning under different objectives. Furthermore, sensitivity analyses and Pareto efficiency experiments are conducted to draw insights regarding the effect of parameter settings and multi-objectives. The computational results demonstrate the efficacy of our developed model and solution approach, tested on a real urban freight transportation network in a major US city.

Keywords: Hybrid modelling, multi-objective optimization, agent-based simulation, physical internet, auction-based mechanism

\section{Introduction}

Freight transportation plays a vital part in moving goods among manufacturers, distributors, retailers, and consumers along regional and global supply chains. Meanwhile, freight transportation planning entails the challenge of adapting to uncertain market changes and increasing customer demands as it involves a complex coordination of process operations and resource allocations. Particularly with e-commerce, businesses 
continue to experience rapid growth. For example, the US Department of Commerce, in its quarterly e-commerce sales report, estimated that retail e-commerce sales for the third quarter of 2019 were $\$ 154.5$ billion, and this was 5\% more than the second quarter of 2019; compared to this, the corresponding increase in total retail sales was only $1.4 \%$ between Q3 and Q2, 2019. ${ }^{1}$ To meet this rise in demand, companies continue to invest in new, expanded and upgraded facilities. In this regard, logistics providers are typically inclined to collaborate vertically along supply chains, focusing on lowering costs and improving efficiency to increase profits. ${ }^{2}$ The technological strides related to innovations in vertical collaborations have led to substantive economic contributions with low-cost international shipping, same-day or on-demand delivery services, and real-time tracking of freight. Vertical collaborations are made possible through a redesign of existing organization structures which improve coordination, decision making, and planning; this prompts increased knowledge sharing and the development of a more flexible and reactive supply chain, better strategic planning, higher vehicle fill rates, reduced transportation costs, increased customer satisfaction, etc. ${ }^{3-4}$ Horizontal collaborations also have environmental benefits. For example, Srinivasan and Leveque report that about one in four trucks in Europe and the US drives empty and, if loaded, it carries just above half of its transport capacity on average. ${ }^{5}$ Sharing can mitigate the issue by higher utilization of vehicles and reducing traffic congestion that directly correlates to an environmental benefit of lowering carbon emissions. Many efforts have been made, for instance, to reduce and control the presence and mobilization of freight vehicles, to eliminate wastes due to a lack of resource sharing, and to improve urban transportation systems. ${ }^{6-7}$

The sharing economy is a peer-to-peer economic model characterized by people and organizations sharing resources. With the advent of the sharing-based economy, an organized collaboration may comprise individual entities working collectively towards a common goal; such collaboration is frequently facilitated through an online platform. Towards this end, the Physical Internet (PI) has been introduced as a potential framework to enable an efficient and sustainable Logistics Web based on physical and operational interconnectivity through the integration of encapsulation, interfaces, and protocols. ${ }^{8}$ Inspired by its digital counterpart (the Internet) and its ability to route TCP/IP packets through a network of computers and routers, PI promotes horizontal collaboration by facilitating the freight movement via shared facilities and consolidating shipments in a decentralized system. 
This paper investigates horizontal collaboration in freight transportation planning using a hybrid modeling approach that combines multiple methods from operations research and computer simulation. The primary motivation for this work is the dearth of literature on integrated, network-based models. Ferrell et al. present a critical review of collaborations in logistics planning and state that most research in network-based models has been limited to modeling a specific design characteristic of horizontal collaboration, e.g., scheduling of vehicles, pricing or lane exchange. ${ }^{9}$ Studies that considered integrating multiple characteristics demonstrated by supply chain collaborations, e.g., combining routing and scheduling with pricing issues, or combining routing and pricing with facility sharing, remain relatively few. Such integrated quantitative models would better capture the potential gains realized through horizontal freight transportation strategies and justify investments in the underlying business models. Motivated by this observation, we contribute to the literature by developing an integrated model at an operational level (network flow) as well as a strategic level (pricing mechanism). Our aim is to first model the open and shared PI-enabled logistics system, and second, to evaluate the potential of such a system in collaborative freight transportation planning among multiple stakeholders in the supply chain. For this integrated modeling, we propose the combined application of analytical techniques, namely auction-based pricing mechanisms, agentbased simulation, and optimization. Our hybrid modeling methodology leverages the strengths of these individual (and interdisciplinary) techniques. ${ }^{10}$ It provides a solution that best applies to the collaborative freight transportation planning scenario that we present in this paper. The hybrid method is used to model an urban area that has a number of logistics clusters serving as hubs for various transportation networks with multiple warehouses operated by e-commerce businesses.

The overall contribution of this paper is the development of an integrated model for freight transportation planning for horizontal collaborations, which considers the operational-level characteristics of the freight network and the higher-level pricing scheme. Three major points are summarized: First, we design an auction mechanism as the communication protocol between shippers and carriers. In this mechanism, the bidding price is determined as a function of traveling cost and truckload fill rate. Second, we develop an agent-based simulation model, based on a logistics system enabled by PI, to demonstrate the benefit of cooperation among service providers and solve a real urban freight transportation network problem in a simulation optimization framework. Third, we develop a hybrid model that combines the auction mechanism with the simulation 
model to represent the interaction among shippers and carriers, matching transport requests and service vehicles in a transport exchange.

The remainder of the paper is organized as follows. Section 2 reviews the literature on collaborative freight transportation planning and auction mechanism, and provides the relevant simulation background. Section 3 describes the development of a hybrid model that consists of three layers (simulation, auction, and optimization). Our simulation optimization approach is the subject of Section 4. Section 5 presents the computational results of sensitivity analysis and multi-objective experiments. Section 6 is our concluding section, where we discuss the implications of this study and suggest directions for future research.

\section{Related literature}

\subsection{Collaborative freight transportation}

Vertical collaboration has been a vital part of supply chain planning for decades, One of its primary intentions is to increase profits and reduce operational costs. Distinct from vertical collaboration along the supply chain, horizontal collaboration in logistics is a concerted practice among organizations that have parallel relationships at the same echelon of the logistics process, performing similar activities or providing similar services that can benefit economies of scale by cooperation. Some examples of horizontal collaboration in logistics include joint route planning, manufacturers consolidation centers, and purchasing groups. Of late, there has been a shift towards horizontal collaboration logistics where individual logistics providers seek and share economic shipping solutions by bundling volumes across various supply chains. ${ }^{11}$ The proactive sharing of transport is part of the PI initiative, ${ }^{12}$ which is defined as an interconnected transportation network and collaborative freight logistics system. More specifically, the Physical Internet protocol, analogous to the digital Internet, focuses on handling standardized PI-containers on the path through shared hubs in the interconnected logistic networks, resulting in an efficient use of transportation facilities and vehicle capacities as well as lowering greenhouse gas emissions. ${ }^{13-14}$

Collaborative freight transportation planning (CFTP) intends to improve a series of operations and joint decision-making processes on suitable allocation of resources in

Supply Webs. ${ }^{15}$ Transit centers provide freight consolidation and cross-docking functionalities instead of direct peer-to-peer delivery from origin to destination. ${ }^{16}$ Coalition trucks planning extends to reallocation of transport requests among the carriers 
to achieve freight consolidation to maximize profits, ${ }^{17}$ rather than only to minimize individual carrier costs associated with vehicle routings. Several large-scale studies have demonstrated further evidence on the potential of PI to realize substantial gains in efficiency and sustainability. Hakimi et al. ${ }^{18}$ study a fast-moving consumer goods (FMCG) industry in France and indicate that PI significantly improves the transportation efficiency (17\% increase in vehicle fill rate), decrease costs $(30 \%)$, and reduce $\mathrm{CO}_{2}$ emissions (up to $60 \%$ reduction). Meller et al. ${ }^{19}$ estimate that the average distance traveled would decrease by $20-30 \%$ and the inventory at the retailer could be reduced by $33 \%$ in a PI-enabled logistics network.

In contrast with vertical cooperation in supply chains, which has been the focus of academics and practitioners for a long time, we note that the literature on horizontal collaboration in logistics is relatively scarce. A survey by Cruijssen et al. ${ }^{20}$ on potential opportunities and impediments for horizontal cooperation indicated that horizontal collaboration could be realized by assessing the benefits of joint route planning. They assert that the expected outcome would include improving the productivity of core activities and reducing supporting activity costs for logistics service providers. Krajewska et al. ${ }^{21}$ proposed finding vehicle routes for carrier collaboration by solving a multi-depot routing and scheduling problem with time windows while minimizing the total cost to meet customer requests. More recently, Fazili et al. ${ }^{22}$ compared the performance of a collaborative transportation system with those of a convention logistics system and an intermediate hybrid logistic system based on Monte-Carlo simulation within a sequential optimization framework. They reported that the collaborative transportation system reduces truck driving distance and greenhouse gas emissions, whereas container transfers are increased. Gansterer and Hartl provided a structured review of collaborative vehicle routing, including centralized collaborative planning, decentralized planning without auctions, and auction-based decentralized planning. ${ }^{23}$

As discussed in Section 1, this paper adds to the freight transportation literature a unique solution approach that integrates a multi-agent simulation framework in conjunction with an optimization and an auction mechanism. Further, our computational results on the benefits of truck utilization and driving distance reinforce the current literature on PI. 


\subsection{Auction mechanism}

Auctions mandate a bidding process that serves as an exchange of shipping service requests and provisions. As such, in a decentralized freight transportation network, it is worth addressing how to dynamically match requests with offers in each transit center while globally optimizing the transport flow in the network. Solving a centralized vehicle routing problem alone is not sufficient for efficient transportation planning with CFTP routing auctions and agent-based simulation (discussed in Section 2.3) would enable the combined application of three well-defined Operations Research techniques through a hybrid modelling framework ${ }^{24}$ for collaborative freight transportation planning. Douma et al. ${ }^{17}$ apply revenue management to agent-based transportation planning, and develop a dynamic programming and approximation approach to price loads in a single-leg problem. Qiao et al. ${ }^{25}$ propose a less-than-truckload dynamic pricing problem in PI where the first-price sealed-bid auction mechanism is adopted and applied in a single-transport problem. Request quantity, carrier capacity, and transportation cost are the factors considered in the optimal pricing decision. Pan et al. ${ }^{26}$ present rules, auction mechanism, as well as bidding and auctioning agents in a simulation framework for transport service allocation in PI.

Exchange mechanisms can be applied in CFTP scenarios to reallocate complementary requests from different carriers. ${ }^{27} \mathrm{~A}$ scheme of horizontal logistics cooperation is applied to find a solution for corresponding pooling games in the study by $\mathrm{Xu}$ et al. ${ }^{28}$ Berger and Bierwirth simplify their exchange mechanism approach by relaxing capacity restriction imposed on a pickup and delivery problem, and compared the costs of no collaboration, collaboration with centralized control, and collaboration with decentralized control. ${ }^{29}$ However, the challenges associated with making information visible across organizations (e.g., inter-organization data sharing and information visibility) and carrier enforcement (e.g., forcing carriers to follow optimal exchange prices) make the approach difficult to consider in practical CFTP scenarios. Pan et al. assert that compared to capacity exchange mechanism, auction mechanism is arguably more suitable to the scope of CFTP, albeit with the aforementioned disadvantages. In general, auction theories can be applied for decision making on choices of, e.g., carriers and hubs. Nevertheless, this approach has not been used and validated by PI network structures using real data. To this end, our study demonstrates the efficacy 
of an auction-based simulation approach to PI logistics and provides accurate performance assessments of the system.

\subsection{Simulation and simulation optimization}

Computer modelling and simulation $(M \& S)$ refers to a computer model of a system of interest, which could be either an existing/real-world system or an imaginary/future system, and its execution over digital computers through a series of experiments developed to help better understand a complex system. The ability of simulation to replicate system behaviors and interactions among modeling components makes it an attractive tool for performance assessments, inferences, as well as predictions. ${ }^{30}$ Given that simulation requires suitable data inputs and provides observations of corresponding outputs, ${ }^{31}$ it serves a host of other purposes such as performing tasks with any potential risk, experiencing certain scenarios in advance, verifying an existence proof, or discovering new and unexpected relationships.

As an analytical decision support tool, computer simulation can be largely categorized as discrete event simulation (DES), system dynamics (SD), agent-based simulation (ABS), or a hybrid of these techniques (e.g., DES and SD, ABS and DES). ${ }^{24}$ Each simulation method is known to effectively target a set of particular type of problems. A DES model represents the system whose state changes occur at discrete points of time, and focus on evaluating the expected performance measures under uncertainty, whereas SD modeling is more suitable for understanding the nonlinear behavior of complex systems using stocks and flows (often in conjunction with differential equations), particularly the state changes occur continuously over time. In comparison with the previous two approaches, ABS is useful to model a system from the agents' point of view and enables modeling heterogeneous and autonomous agents acting independently in the environment as well as the emergence of self-organization. ${ }^{33}$ (See Section 3.1.1.)

The continuing strides in computer technology, both in terms of hardware and software, has immensely contributed to the field of M\&S. It has led to the development of increasingly complex models and has enabled faster execution of simulations. Simulation has been recognized as one of the most frequently used OR techniques and used as a decision-making tool, to model complex real-world systems, estimate performance measures, and find optimal responses. Simulation optimization is a process of search for the best input configuration without evaluating every possibility. ${ }^{34}$ While simulation optimization seeks to obtain information relevant to an objective function 
using minimum resources, it is more than merely enumerating a finite number of experiments and considering some inputs as a selection of configurations. Rather, simulation optimization techniques dictate the sequence of experiments in order to calculate the best input factors within an acceptable tolerance or a time limit. ${ }^{35}$

Due to the nature of the stopping criteria in simulation runs and the inefficiency of exhausting all possible input combinations, a global optimum is not guaranteed. However, heuristic algorithms can be used to overcome such barriers as stalling at a local optimum by searching over a wide solution space. ${ }^{36}$ Using these procedures, the optimization strategy takes the output of a validated simulation model, provides feedback on the progress toward achieving a different input parameter setting, then adjusts the inputs as necessary to improve the output. Simulation optimization methods are broadly classified into six major categories: gradient-based search methods; stochastic optimization; response surface methodology; metaheuristics; A-Teams; and statistical methods. For example, statistical procedures include ranking and selection and multiple comparison. More specifically, multiple comparison techniques provide an ordinal ranking of simulation configurations, while ranking and selection techniques indicate a magnitude of measurement to distinguish between configurations.

Banks et al. ${ }^{37}$ break down the approaches toward simulation optimization into four categories: asymptotic convergence to the optimum; optimality under deterministic counterpart (mathematical programming); a prespecified probability of correct selection (from a set of alternatives); robust heuristics (combinatorial search algorithms). In the literature, there are also several comprehensive survey papers that discuss foundations, theoretical developments, as well as applications of a variety of techniques and approaches. $^{38}$

\section{Hybrid modeling framework}

Hybrid modelling (HM) is a combined application of simulation with methods and techniques from disciplines such as applied computing, computer science/applied computing, engineering, data science, and operations research/management science. Compared to hybrid simulation (HS), which refers to the combined application of discrete and continuous simulation approaches such as SD, DES, and ABS to a single simulation study (see Brailsford et al. ${ }^{39}$ for a recent review of literature on this topic). HM is broader in scope. HM advocates the use of cross-disciplinary approaches, including interdisciplinary research paradigms, frameworks, methodologies, techniques, and tools 
to one or more stages of a simulation study (e.g., conceptual modeling, model implementation, model execution, scenario development, etc.) with the objective of creating the best possible representation of the system of interest. ${ }^{40}$ Mustafee and Powell in their paper ${ }^{24}$ titled "From Hybrid Simulation to Hybrid Systems Modelling" outline the difference between HS and HM approaches and present a unifying HS-HM conceptual representation. This unification includes four types of hybrid models (Types A to D). Types A to $\mathrm{C}$ are the various forms of HS.

- Type A (multi-methodology HS) is a combined application of continuous and discrete simulation approaches (e.g., SD and ABS);

- Type B (multi-technique HS) is a combined application of two discrete simulation approaches, e.g., ABS and DES.

- Type C is multi-methodology, multi-technique HS, which is both Type A and Type B. For example, the combined application of ABS, DES, and SD.

- Type D is HM, which is a combined application of computer simulation approaches (SD, DES, ABS) with methods and techniques from the broader Operations Research/Management Science (ORMS) discipline.

The hybrid model that we present in this paper is of Type D. The overarching HM is realized through a combined application of auction mechanisms (used frequently in Economics) with an ABS snf optimization approach. Why is an integrated approach necessary? Ferrell et al. outline that a need for integrated quantitative models would better capture the potential gains that can be realized through horizontal freight transportation strategies. ${ }^{9}$ In our study, we implement a hybrid model comprising an agent-based simulation of a decentralized transport network in level one (simulation level; Section 3.1.1), an auction mechanism to determine a winner in the bidding process for shipping services in level two (auction level; Section 3.1.2), and an optimal search process in level three (optimization level; Section 3.1.3).

\subsection{Multi-layer structure}

The structure of our model is layered in three levels of hierarchy from simulation to auction and to optimization (Figure 1). We develop an agent-based model using the simulation software AnyLogic to represent an interconnected urban logistics system. 


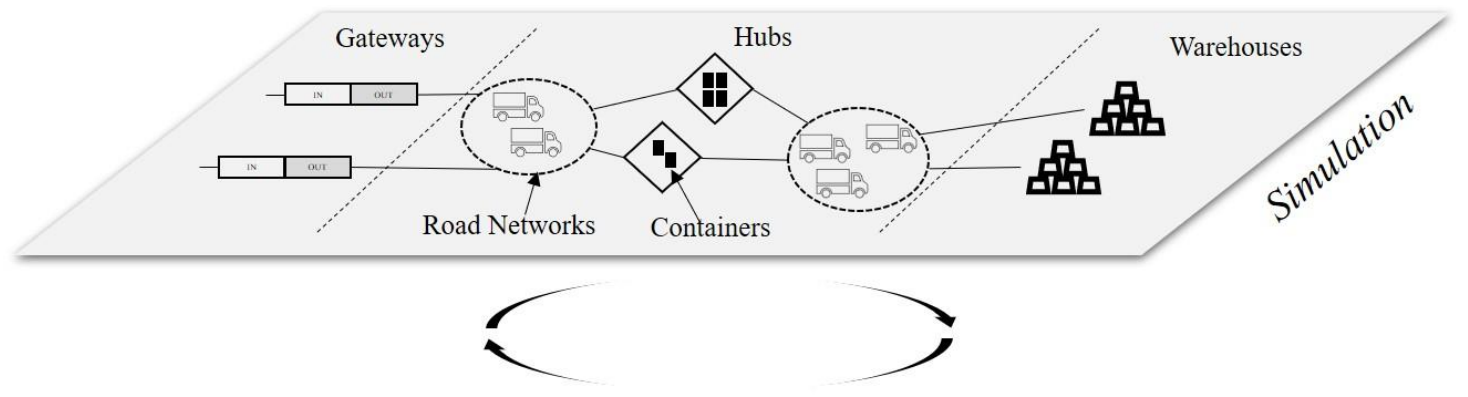

Carriers: Bidding price function \{cost, truckload fill rate, profit margin\}

Container's request: payments

Determination

Program

$\lambda^{u^{c}}$

- Decision variable and parameter settings

- Optimisation via simulation

- Sensitivity analysis and pareto efficiency
Single\&

Multiple

Objectives
OP

Figure 1. A hybrid model consisting of three levels (simulation, auction, and optimization).

\subsubsection{Simulation level}

Computer-based simulation modeling has been widely used to gain insights and conduct analysis, by explicating complex system behaviors and stochastic interactions among components in the logistics and transportation domains. ${ }^{41} \mathrm{ABS}$ aids to model individual agents their decision-making behaviors and rules. By observing the effects of agent attributes and interactions, $\mathrm{ABS}$ offers flexibility to understand the system as a whole and elicit emerging system-wide changes. In an agent-based modeling framework of logistics, large-scale complex interacting nodes, facilities, transport entities or even decision makers, as part of a distributed system, work intelligently at a local level. ${ }^{31}$ Using ABS, Serrano-Hernandez et al. address the evolution of horizontal cooperation over time and also its impact on costs and $\mathrm{CO}_{2}$ emissions, ${ }^{42}$ and Sarraj et al. propose and test a multi-agent simulation model to assess the potential of using PI protocols that can improve a transportation system. ${ }^{14}$

Our developed model is decentralized in a simulated environment using geographic information system (GIS) where trucks deliver containers through a transport 
network with hubs. The model places warehouses at the locations of e-commerce companies and generate an outbound flow of goods. Freights from outside the boundary are transported through gateways into the city. Hubs are mainly located close to the intersections of major highways and warehouse-dense areas, thereby reducing the times required for consolidation of shipments. When freight instances occur in the urban logistics system, they are either distributed to gateways or transhipped to other transportation nodes for outbound shipment. Representing a basic unit of freights in the system, the model generates containers at warehouses and from gateways, and eventually leave the system when they reach their destinations. Trucks pick up containers and deliver directly to destinations or move to hubs for a further consolidation of shipments.

\subsubsection{Auction level}

In a collaborative transportation system, shippers benefit from selecting their carriers, and the allocation of shipping services to a designated carrier becomes important. The communication between shippers and carriers follows auction-based principles by matching transport requests with services. When a container needs transportation, it sends a request to available trucks and selects one based on the auction criteria. To achieve revenue maximization for carriers and cost minimization for shippers, we propose a bidding process to allocate shippers to carriers, who are acting as bidders and auctioneers, respectively. The simulation model then calls an optimization subroutine as a truckselection method in each bidding process. Within such a framework, the logistics information of each carrier (e.g., cost rate, revenue expectation, and capacity) is also considered, and subsequently, these factors serve as parameters in the next optimization level.

Figure 2 illustrates the improvement in carrier transportation routes and fulfilments after an exchange of containers in Hub 3 between Carriers 1 and Carriers 2 . Carrier 1 starts from $\mathrm{Hub} 1$ with three containers on board, whose destinations are $\mathrm{Hubs}$ 3, 4, and 5, respectively. Along its route, Carrier 1 stops at Hub 3 to deliver one container and to pick up one additional container for $\mathrm{Hub}$ 5. Carrier 1 then stops at $\mathrm{Hub} 4$ to offload one container, and finally it delivers the last two containers to Hub 5. Similarly, Carrier 2 starts at Hub 2 with three containers and delivers them to Hubs 4 and 5, respectively. Figure 2(b) shows an exchange at Hub 3 by switching a container meant for Hub 5 (in black) from Carrier 2 to Carrier 1, as well as a container heading for $\mathrm{Hub} 4$ (in white) from Carrier 1 to Carrier 2. Hence, Figure 2(b) presents a better scenario of increasing 
the utilization of truckload and saving travel distance. This method amplifies the efficiency of transporting goods by further utilizing a coalition of carrier vehicles.

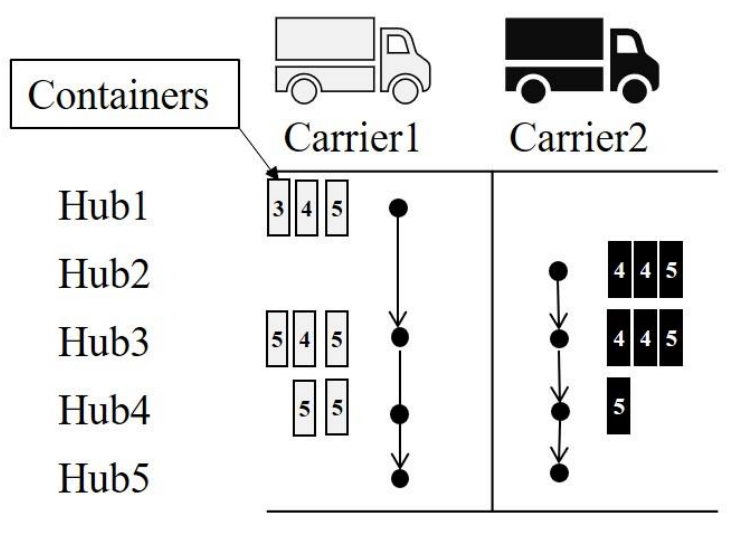

(a)

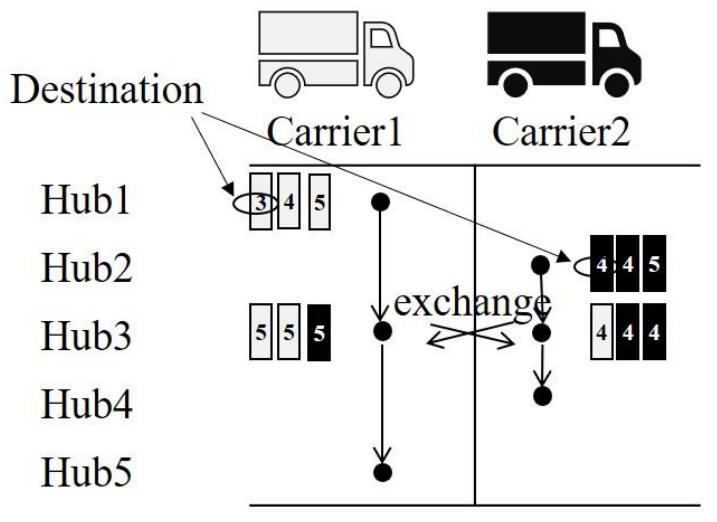

(b)

Figure 2. Routing plan before (a) and after (b) exchange (switching) of containers between Carrier 1 and Carrier 2 at $\mathrm{Hub} 3$.

\subsubsection{Optimization level}

A simulation optimization approach is employed in this study to determine the most relevant decision variables for transportation planning in a stochastic system. By simulating various multiple scenarios where input decisions change and randomized samplings are required, the model identifies the best case based on performance measures by comparing objective values.

In search of improved objective values, an optimization module is integrated with the simulation software used in this study. The module employs a combination of strategies based on scatter search and tabu search, combined with configuring and training neural networks for screening out candidate solutions likely to perform poorly. More specifically, similar to genetic algorithms, scatter search is a population-based procedure that generates offspring by incorporating past evaluations. These new candidate solutions produced by metaheuristics are passed on for evaluation in iteration. Subsequently, the neural network works as a screening mechanism to eliminate particular candidates whose objective function values are predicted to "not improve" the current solution (no additional simulation runs are performed to determine these candidate solutions as neural networks are being used for inductive learning). The screening decision is made based on an amount exceeding the risk level, i.e., deviations from the best solution found up to that point. Moreover, we formulate and solve optimization problems as single or multi- 
objective models according to key performance metrics related to time and cost. This is further discussed in detail in Section 4.

\subsection{Agent representation}

This section describes three key agent classes that are the building blocks of the ABS model, namely the container agent, the truck agent, and the hub agent.

\subsubsection{Container agent}

A container agent represents a basic unit of freight shipped within the capacity of a truck. In the context of PI, our model assumes that containers are of a standard size in volume when estimating truck shipment loads. This differs from the hybrid ABS-CLS study by Mustafee and Bischoff where containers have verying dimensions and load-bearing capacity. ${ }^{43}$ Figure 3 depicts a logic of state flows of a container agent. Container agents are created from warehouses and other gateways, and the shortest route is determined for the container to pass through a network of hubs, and the container exits the system when it reaches a destination. The state of LookForATruck indicates that a container sends a request for a current leg of its trip and selects a preferred truck. Then the container waits to be picked up by a truck (state: WaitForTheTruck), and finally the container is loaded on the truck (state: InTruck). When the container arrives at a hub, it is sorted for its next ride while seeking an available truck if necessary. If a current stop location is the container's final destination, its trip ends and the container is removed. This procedure repeats until all containers reach their final destinations. 


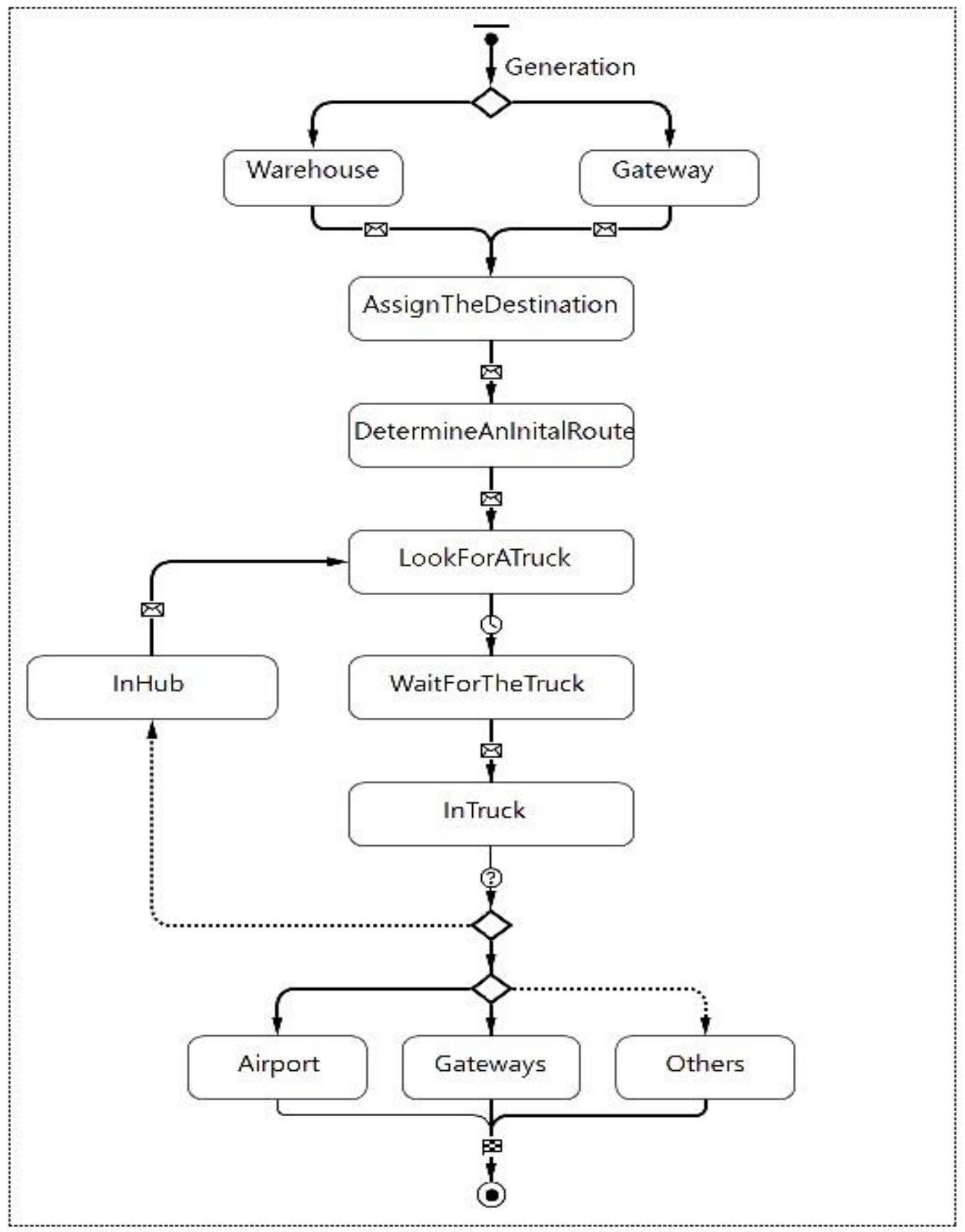

Figure 3. State flows of a container agent.

\subsubsection{Truck agent}

Figure 4 depicts the process of a truck that transports and responds to the requests from containers. All instances of a truck agent are initially generated at the start of a simulation run. In the state WaitForARideRequest, truck agents are idle with an empty schedule. Once a ride request is accepted and entered in the schedule, the agent changes its state to Loading and WaitForContainers. Trucks respond to the transport requests of containers, and make a sequence of rides. Note that a truck agent adds a container to a scheduled ride 
only if additional capacity is available. A truck undocks (state: Undocking) from the current hub and leaves for a next stop within a specific time window. After completing a ride to a hub (this is after states: Moving, Docking, Unloading), the truck can bid on the auction for a next ride. For a modeling purpose, it is assumed that all truck agents have the same volume capacity and move at a constant speed.

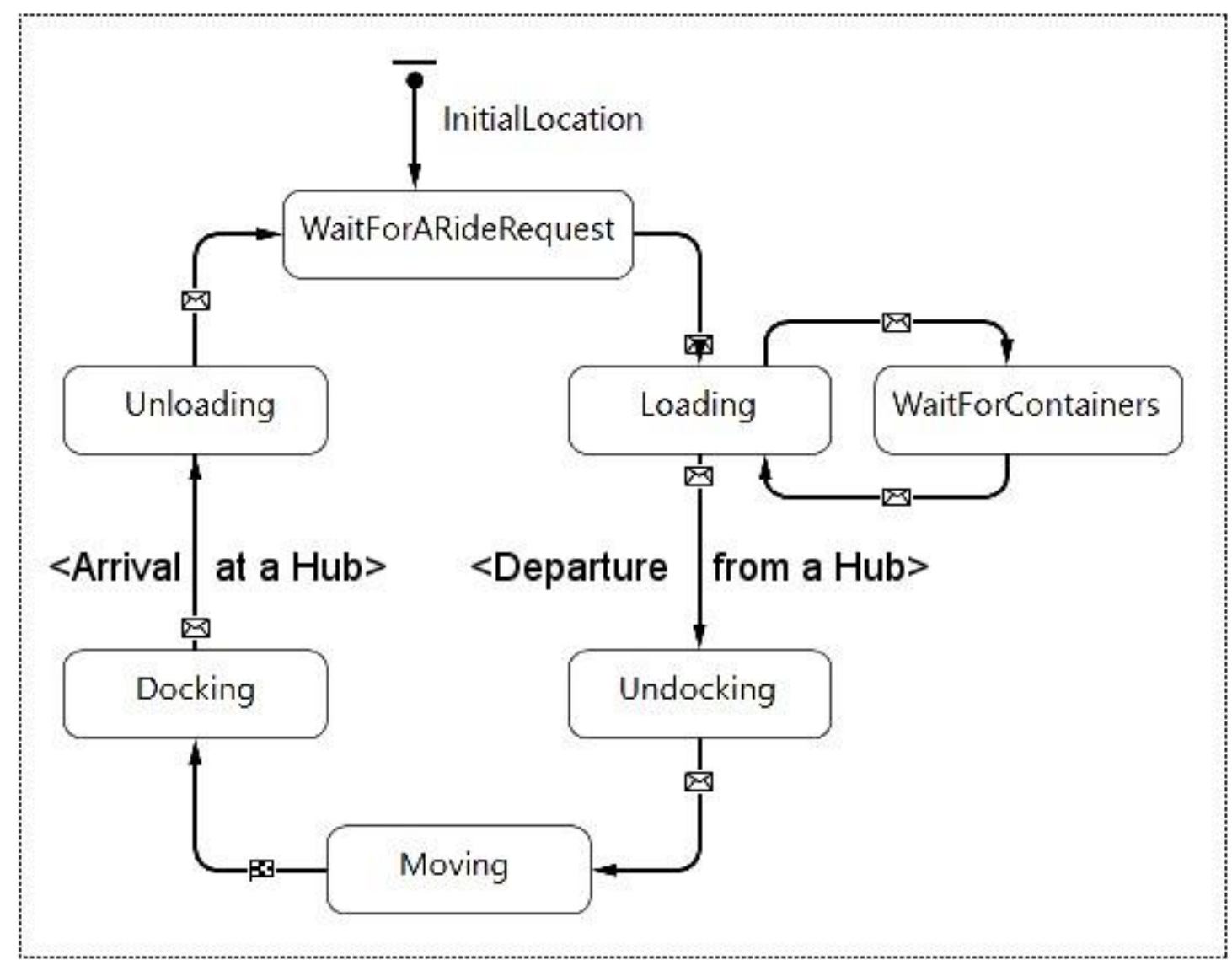

Figure 4. State flows of a truck agent.

\subsubsection{Hub agent}

Hub agents are placed in the system as an input to the simulation model. They can be in any one of the following seven states shown in Figure 5, namely, DockTrucks (dock a truck in a loading bay); UnloadTrucks (unload a truck and receive containers); InHub (let a truck wait in a loading bay); SortContainers (sort arrived containers for their next ride); WaitForPickup (let containers wait for pickup by an assigned truck); InTruck (load a truck with the assigned containers); and UnDock (undock a truck from a loading bay and release from the hub). 


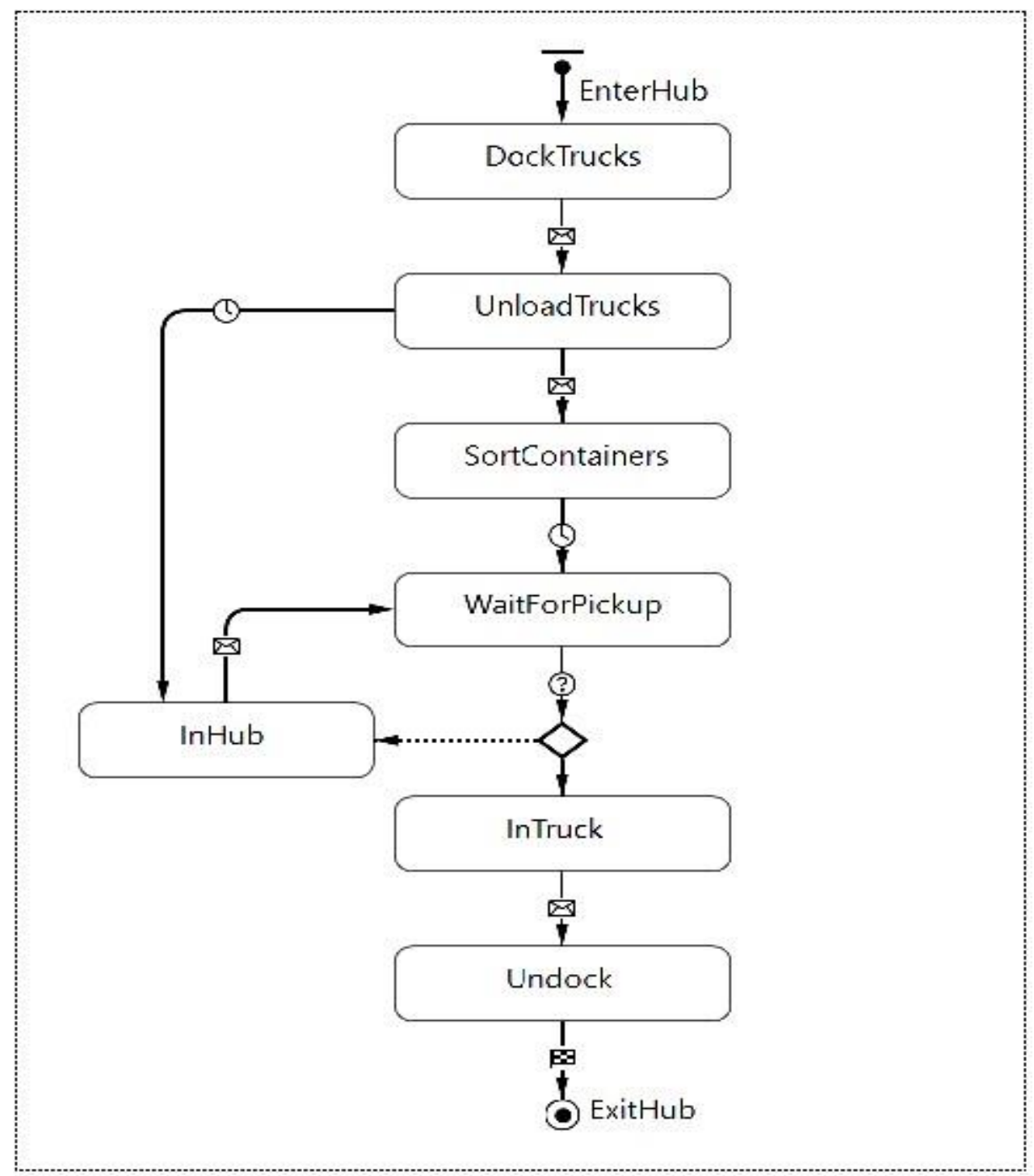

Figure 5. State flows of a hub agent.

Each PI-hub serves as not just a loading or transhipment facility but also as a service point where containerized shipments are reallocated to a more efficient way at lower price. Section 3.3 takes a closer look at the auction process that allows for such modeling capability.

\subsection{Auction process}

A goal of CFTP is to maximize carrier's joint profits while meeting delivery requests under a framework of collaborative fulfilments. In other words, it is to achieve synergistic benefits by a coalition of LTL (less than truckload) carriers rather than maximizing individual benefits. Agent-based transportation planning takes advantage of decentralized operations and dynamic processes. Figure 6 shows its structure pertaining to the auction 
mechanism. Shippers send out transport requests, and carriers bid on them in an auction where travel cost factors are considered in (re)assigning a container to a truck at hubs under a given time window. The auction process considers the remaining capacities of a truck, i.e., the truckload utilization, when determining a winner of most cost savings.

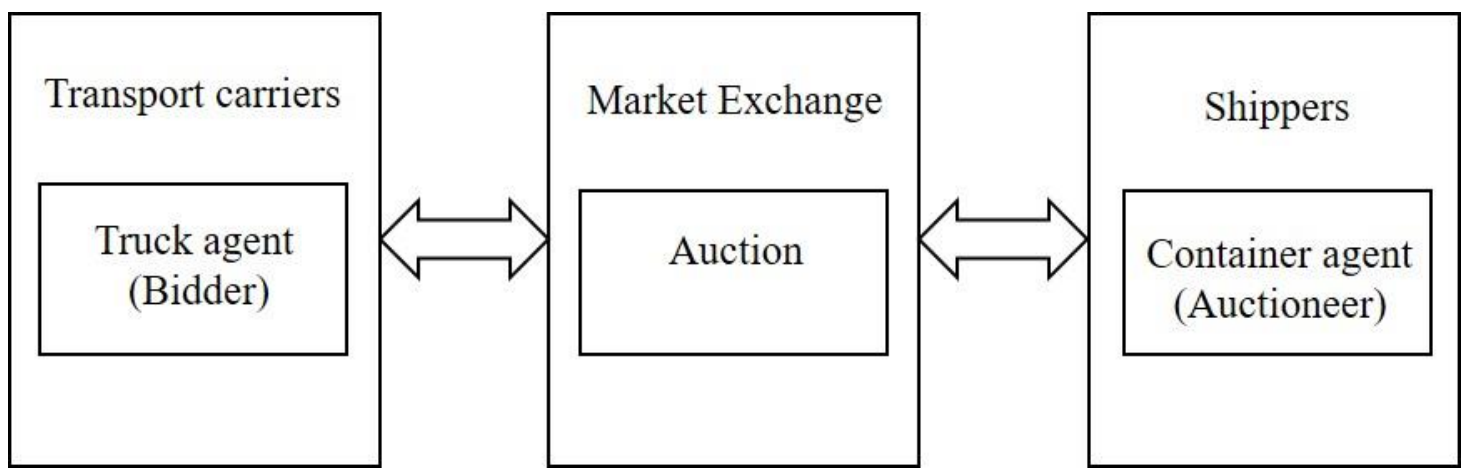

Figure 6. In a PI transportation system, relationships between carriers and shippers are established through an auction mechanism.

We design an auction protocol to include bidders and auctioneers, represented as trucks (carriers) and containers (shippers), respectively. A carrier makes a bid for each feasible request with a bidding price based on transport cost and expected profits. A shipper takes the role of an auctioneer and facilitates container allocations and route selections while satisfying carrier capacity. Table 1 shows a procedure of a stop-by-stop auction scheme; containers stop at hubs and initiate an auction at each stop.

Table 1. An algorithmic procedure of auction exchange.

\section{Step 1. (Initial routing)}

Set the route for each container at its origin.

\section{Step 2. (Requesting)}

At each stop, the container can make a request for auctioning.

\section{Step 3. (Bidding and auctioneering)}

Carriers propose their bidding price; shippers consider these bids in a pool of auction.

\section{Step 4. (Incentive)}

If the same truck wins a bid, then the associated setup costs are waived.

\section{Step 5. (Assignment)}

Assign the container based on a winner determination program.

Else, go to Step 2. 
Step 1 provides initial routing by finding the shortest path of a container at the point in time when it is first generated in the network. A ride is transporting of containers (by a truck) from one stop to another (these stops are also known as hubs). A route refers to the path of a container with a sequence of stops that include an origin and a destination. We use the Dijkstra's shortest path algorithm to generate the initial node sequences between departure and arrival hubs along the path.

In Step 2, shippers make transport requests that comprise a selection pool in the auction for carriers. Compatible requests are grouped along the portion of the route in common. Prior to describing the auction model, we first define the notations as follows.

\section{Notations:}

$T$ Set of total trucks in the auction, indexed by $t$.

$R$ Set of total requests in the auction, indexed by $r$.

$R_{t}$ Set of requests that can be served by a truck $t \in T, R_{t} \subseteq R$.

$d_{r}$ Distance of a request $r \in R$.

$n_{r} \quad$ Number of containers of a request $r \in R$

$f_{t} \quad$ Load fill rate at bidding by a truck $t \in T$.

$c_{f}^{t} \quad$ Fixed/processing cost of a truck $t \in T$.

$c_{v}^{t} \quad$ Distance-volume based variable cost of a truck $t \in T$.

$T C_{r}^{t}$ Transportation cost of a truck $t$ for a request $r \in R_{t}, t \in T$.

$P_{r}^{t} \quad$ Bidding price by a truck $t$ for a request $r \in R_{t}, t \in T$.

$P A_{r}^{t}$ Payment to a truck $t$ by a request $r \in R_{t}, t \in T$.

Once requests are generated, Step 3 populates bidding prices for each request by auctioneers. The transportation cost of truck $t$ for request $r, T C_{r}^{t}$, is defined in Equation (1).

$$
T C_{r}^{t}=c_{f}^{t}+c_{v}^{t}\left(1-f_{t}\right) d_{r} n_{r}, \forall r \in R_{t}, t \in T
$$

where the transportation cost consists of fixed cost and variable cost associated with the current truckload utilization, travel distance, and number of containers for the request. Based on the cost function and an expected profit factor, a bidding price by the carrier for a request $r, P_{r}^{t}$, is set by Equation (2).

$$
P_{r}^{t}=T C_{r}^{t}\left(1+m^{t}\right)
$$

where $m$ is a margin rate of bidding price by carrier $t$.

In Step 4, shippers determine the payment to carriers, which is defined for a given request $r$ as $P A_{r}^{t}$ in Equation (3). 


$$
P A_{r}^{t}= \begin{cases}P_{r}^{t}-c_{f}^{t}, & \text { if a container stays the same truck } \\ P_{r}^{t}, & \text { otherwise }\end{cases}
$$

where the processing $\cos t c_{f}^{t}$ incurs at a hub. If a truck wins the bid for the same shipment consecutively, it incentivizes a continuity of shipments by waiving the associated processing cost.

The winner determination program (WDP) selects the winning bid in Step $5 .{ }^{21} \mathrm{In}$ the case where no winner can be determined in the auction process, a container waits for a given time limit, and then reverts to the Step 2. A WDP problem is formulated as follows:

$$
\begin{array}{ll}
\text { Minimize } & \sum_{t \in T} \sum_{r \in R} P A_{r}^{t} x_{r}^{t} \\
\text { subject to } & \\
& \sum_{t \in T} x_{r}^{t}=1, \forall r \in R \\
& \sum_{r \in R} x_{r}^{t} \leq 1, \forall t \in T \\
& x_{r}^{t} \in\{0,1\}, \forall t \in T, \forall r \in R
\end{array}
$$

where the objective function (4) minimizes a total cost for allocating all requests. Constraint (5) assigns each request to exactly one truck, while Constraint (6) ensures that each carrier will win at most one request. $x_{r}^{t}$ is a binary decision variable with its value of one if request $r$ is allocated to carrier $t$, and zero otherwise in Constraint (7).

The WDP is solved using a Java package of IBM ILOG CPLEX that includes an optimization routine called by the simulation model. We take into consideration feasible request constraints in the simulation process module including time window constraint, truckload capacity, and travel speed. While a winner to each request is optimally determined during the simulation run, system-wide performance metrics are measured by a simulation-optimization approach as discussed in Section 4.

\section{Simulation optimization approach}

Multiple inputs of logistics system components have variable effects on the model outcomes concerning efficiency and sustainability. This includes parameters such as fixed and variable costs, arrival rates of containers, time-window constraints, as well as decision variables (e.g., truck capacity). To find a set of decision values that result in optimality, we take an optimization via simulation approach using OptQuest, which guides the search path for optimal solutions in the simulation model. 


\subsection{Model parameters and variables}

Parameters related to containers include arrival rates at gateways and warehouses. Truckrelated parameters include the number of trucks, truck capacity, truck speed, and a maximum wait time. Parameters associated with hubs include docking time, loading time, and sorting time. Table 2 shows a list of parameters along with their initial values. We assume that the indicative values of cost parameters are based on the estimation of shares of all containers in the PI system. The variable cost consists of fuel cost and other externality costs, while the fixed/setup cost involves sorting and (un)loading costs.

Table 2. Parameters and initial values.

\begin{tabular}{ll}
\hline Parameter & Value \\
\hline Truck speed & $50 \mathrm{~km} / \mathrm{h}$ \\
Maximum wait time & 2 hours \\
Dock time & 5 minutes \\
Load time & 5 minutes \\
Sort time & 15 minutes \\
Arrival rate in warehouses & 1.8 containers/hour \\
Arrival rate in gateways & 18 containers/hour \\
Variable cost rate & $\$ 1 / \mathrm{km}$-container \\
Fixed /setup cost & $\$ 3 /$ container \\
Profit margin & $15 \%$ \\
Number of warehouses & 26 \\
Number of gateways & 5 \\
Number of hubs & 13 \\
Number of airport & 1 \\
\hline
\end{tabular}

The lower and upper bounds on decision variables are set while taking discrete integer values in ranges of $5 \leq$ truck capacity $\leq 10$ and $25 \leq$ number of trucks $\leq 50$. These restrictions are imposed to reduce the search domain of the solution space and thereby find an optimal solution in reasonable times.

\subsection{Objectives}

A cost-associated objective is most common in freight transportation planning and is the main focus of this study. Additionally, time- and environment-driven objectives are also 
considered. This section describes three individual objectives (Objectives 1-3) as performance measures of interest.

To maximize economic savings, the first objective is conversely to minimize the average truck distance per container delivered as in Equation (8).

Objective 1: $\quad \operatorname{Min}_{D}\left\{\frac{\text { total truck distance }}{\text { total number of containers shipped }}\right\}=\frac{\sum_{t \in T} b_{t}}{\sum_{t \in T} n_{t}^{c}}$

where $D$ is the decision space of variables in the system, i.e., truck capacity and truck numbers. $b_{t}$ is the travel distance of truck $t$, and $n_{t}^{c}$ is the number of containers shipped by truck $t$.

The next objective is to minimize the average truck lead time per container and calculated as total truck lead time divided by the number of containers shipped.

$$
\text { Objective 2: } \quad \operatorname{Min}_{D}\left\{\frac{\text { total truck lead time }}{\text { total number of containers shipped }}\right\}=\frac{\sum_{t \in T} l_{t}}{\sum_{t \in T} n_{t}^{c}}
$$

where $l_{t}$ is the lead time of truck $t$.

The objective of environmental sustainability is formulated as the number of containers shipped divided by the total amount of truck space.

$$
\text { Objective 3: } \quad \operatorname{Max}_{D}\left\{\frac{\text { total truckload }}{\text { total amount of truck space }}\right\}=\frac{\sum_{t \in T} n_{t}^{c}}{n_{t} c a p_{t}}
$$

where $n_{t}$ is the number of trucks and $\operatorname{cap}_{t}$ is the capacity of truck $t$ in terms of containers.

We also investigate multi-objective problems by considering two or three objectives concurrently. Objectives 4-6 in Table 3 are bi-objectives that considers any two of Objective 1-3, whereas Objective 7 combines all three objectives. The multiobjective optimization utilizes weights to find the Pareto front in the trade space. For example, $\omega, \lambda, \theta, \varphi \in[0,1]$ control weight distributions to respective Objectives 4-7.

Table 3. Formulations of bi/multi-objectives.

\begin{tabular}{cl}
\hline Objective & Formulation \\
\hline 4 & $\operatorname{Min}_{D}\left\{\sum \omega *\right.$ travel cost $+(1-\omega) *($ lead time $\left.)\right\}$ \\
5 & $\operatorname{Min}_{D}\left\{\sum \lambda *\right.$ travel cost $-(1-\lambda) *($ truckload utilization $\left.)\right\}$ \\
6 & $\operatorname{Min}_{D}\left\{\sum \theta *\right.$ lead time $-(1-\theta) *($ truckload utilization $\left.)\right\}$ \\
7 & $\operatorname{Min}_{D}\left\{\sum \varphi_{1} *\right.$ travel cost $+\varphi_{2} *$ lead time $-\left(1-\varphi_{1}-\varphi_{2}\right) *$ \\
& $($ truckload utilization $)\}$ \\
\hline
\end{tabular}




\section{Computational experiments}

For simulation runs, we determine the number of replications required to limit a relative error $\beta$ using the following approximation of Equation (11).

$$
n_{r}(\beta)=\min \left\{i \geq n_{r}: \delta=\frac{t_{i-1,1-\alpha / 2} \sqrt{S^{2}\left(n_{0}\right) / i}}{\left|\bar{X}\left(n_{0}\right)\right|} \leq \beta^{\prime}\right\}
$$

where $i$ is the number of replications subject to $\beta$ and $\beta^{\prime}=\beta /(1+\beta)$ is the adjusted relative error threshold. With $\beta=0.05$ (or $\beta^{\prime} \approx 0.048$ ) and a confidence interval of $95 \%$, ten replications $(i=10)$ suffice to contain the value of $\delta$ no more than $\beta^{\prime}$. Table 4 provides the relevant statistics. In addition, a batch means method is used to find a warm-up period required to reach steady states. The mean value of the warm-up period over ten replications is two days, and we estimate performance metrics after determining the length of the warm-up period, the length of a batch, and the number of batches.

Table 4. Sample means and variances with ten replications.

\begin{tabular}{llll}
\hline performance measures & $\bar{X}$ (sample mean) & $S^{2}$ (sample variance) & $\delta$ \\
\hline $\begin{array}{l}\text { average truck distance per } \\
\text { container }\end{array}$ & $13.7 \mathrm{~km}$ & 0.520 & 0.038 \\
$\begin{array}{l}\text { average lead time of a } \\
\text { container }\end{array}$ & 3.5 hour & 0.039 & 0.040 \\
\hline
\end{tabular}

The model is implemented using a real urban transport network that includes the outbound and transhipment flows of 26 e-commerce warehouses in Louisville, Kentucky in the US. For model validation, the output performance measures are compared on truck distance per container and average lead time per container with the statistics collected from actual data as baseline (Table 5). The baseline scenario with a selection method of "closest truck first" achieves the minimum average truck distance per container, while the other scenario with an "earliest truck first" selection method attains the minimum average lead time per container. The performance of the proposed model is comparable between these two baseline scenarios. It is noted that the CFTP values in both metrics are relatively close (within a 3\% difference) to those resulting from the baseline scenario with a closeness selection method. This is due, in part, to the bidding price being set considering the cost associated with travel distance rather than travel time.

Table 5. Copmarison of performance measures comparison between the two baseline scenarios and the proposed CFTP model. 


\begin{tabular}{cccccc}
\hline \multirow{2}{*}{ Scenario } & Selection method & $\begin{array}{c}\text { Average truck } \\
\text { distance per } \\
\text { container }(\mathrm{km})\end{array}$ & $\begin{array}{c}\text { Gap } \\
\text { percent } \\
\text { relative } \\
\text { to CFTP }\end{array}$ & $\begin{array}{c}\text { Average lead } \\
\text { time of a } \\
\text { container } \\
\text { (hour) }\end{array}$ & $\begin{array}{c}\text { Gap } \\
\text { percent } \\
\text { relative to } \\
\text { CFTP }\end{array}$ \\
\hline Baseline & Closest truck first & 13.4 & $-2.2 \%$ & 3.6 & $2.9 \%$ \\
\cline { 2 - 6 } & Earliest truck first & 14.2 & $3.6 \%$ & 3.2 & $-8.6 \%$ \\
\hline CFTP & Auction mechanism & 13.7 & - & 3.5 & - \\
\hline
\end{tabular}

In addition, to assess the robustness of our proposed CFTP model involving PI, we consider varying the input parameter values of the variable cost and the number of warehouses by taking into account changes in gas price and supply chain infrastructure, respectively. In Table 6, when either of the two input variables is changed, there is little or no impact on average truck utilization for CFTP which is directly related to the shipping demand and capacity supply. On the other hand, the average lead time for CFTP decreases by $9.4 \%$ and $2.5 \%$ when the number of warehouses in the simulation model increases from 18 to 26 and further from 26 to 34, respectively. Having additional warehouses can accommodate more trucks bidding close to the point where the shipping demand occurs. This means a reduction in wait time for a package, which contributes to a decrease in the total lead times overall. Furthermore, as the variable cost increases from $\$ 0.5$ to $\$ 1$ and to $\$ 2$, the average lead time slightly decreases by one to three percentage points. This is due in part to the cost structure that incentivize keeping the same packages in the same vehicle through warehouse exchange locations, which is all the more attractive when the variable cost rises.

Table 6. Comparison of baseline and CFTP regarding driving time and truck utilization corresponding to change of the input parameter.

\begin{tabular}{|c|c|c|c|c|c|}
\hline & & \multicolumn{2}{|c|}{ Average driving time $(\mathrm{hr})$} & \multicolumn{2}{|c|}{ Average truck utilization } \\
\hline & & Baseline & CFTP & Baseline & CFTP \\
\hline \multirow{3}{*}{$\begin{array}{l}\text { Number of } \\
\text { warehouses }\end{array}$} & 18 & 0.95 & 0.83 & $58.7 \%$ & $77.3 \%$ \\
\hline & 26 & 0.89 & 0.81 & $61.5 \%$ & $77.4 \%$ \\
\hline & 34 & 0.82 & 0.74 & $63.1 \%$ & $77.7 \%$ \\
\hline \multirow{3}{*}{$\begin{array}{c}\text { Variable } \\
\text { cost }(\$ / k m- \\
\text { container) }\end{array}$} & 0.5 & 0.89 & 0.82 & $61.9 \%$ & $77.2 \%$ \\
\hline & 1 & 0.88 & 0.81 & $62.5 \%$ & $77.4 \%$ \\
\hline & 2 & 0.84 & 0.79 & $64.8 \%$ & $77.4 \%$ \\
\hline
\end{tabular}


The following section (Section 5.1) uses sensitivity analysis to demonstrate the fidelity of our model in relation to each performance measure. Section 5.2 presents experiments in the search of Pareto frontiers with the multi-objectives listed in Table 3.

\subsection{Sensitivity analysis}

Sensitivity analyses are conducted to assess the effects of individual parameters on each of Objectives 1-3. The experiments are set up by changing one parameter at a time while keeping the other parameters unchanged. We use forward differencing to compute the changes in the objective function value when increasing one percent of a selected parameter.

The solution to Objective 1 of the average truck distance per container is $D$ (truck capacity, truck numbers $)=[9,27]$ and the objective value is $1,264.73$. Figure 7 illustrates the result of a one-way sensitivity analysis of cost-related Objective 1. Most of the parameters have substantial impacts on the objective function value. Profit margin plays an inhibiting role in the minimization process with a $0.95 \%$ increase of objective function value, so do variable cost and fixed cost by $0.65 \%$ and $0.46 \%$, respectively. Bidding price rises due to the increase of cost factors and profit margin, which leads to less collaborating shipments but more individual direct shipments, thereby increasing the overall travel distance. In contrast, the other parameters enhance the collaboration and contribute to reducing the objective value, particularly maximum wait time $(-1.43 \%)$, arrival rate in warehouses (-1.39\%), and arrival rate in gateways (-0.67\%). As a result, the average truck distance per container decreases. Longer maximum wait time and higher truck speed allow carriers to have sufficient time to allocate shipments in the auction. A higher arrival rate increases the number of containers, and subsequently, the number of requests in the bidding process. The arrival rates in warehouses have a relatively more significant impact $(-1.39 \%)$ when compared to arrival rates in gateways due to the higher truckload fill rates during pickup in warehouses.

The solution to Objective 2 of the average truck lead time per container is $D$ (truck capacity, truck numbers $)=[10,50]$ and the optimal objective value is 0.81 hour. Figure 8 shows the result of sensitivity analysis for each of the parameters and their effects on Objective 2. Arrival rates in warehouses and gateways affect the number of containers in the system for a given time of period, resulting in decreases of $0.42 \%$ and $0.33 \%$ on average lead time per container, respectively. However, time-related factors greatly affect the objective value. For example, a $1 \%$ increase of maximum wait time extends the 
average lead time per container by $1.32 \%$, while the same additional amount of sorting time spent in hubs inflates by as much as $1.04 \%$. Cost-related factors including profit margin, variable cost, and fixed cost, have little impact on Objective 2.

The solution to Objective 3 of the average truckload utilization is $D$ (truck capacity, truck numbers $)=[8,32]$ and the optimal objective value is 0.77 . Figure 9 shows sensitivity analysis for each of the parameters and their effects on the objective. The maximum waiting time allowed in the hub plays an important role in inducing a collaboration of shipments and increasing the truckload utilization by $1.73 \%$. Higher arrival rates of containers from warehouses and gateways are more likely to fill the vacant truck space during pickups and backhauls. On the other hand, increasing profit margin, variable, or fixed cost has a negative effect on Objective 3, resulting in a decrease in truckload utilization by $0.85 \%, 1.20 \%$, and $0.91 \%$, respectively. This decrease in truckload utilization is not surprising as these factors reduce opportunities for collaborating shipments

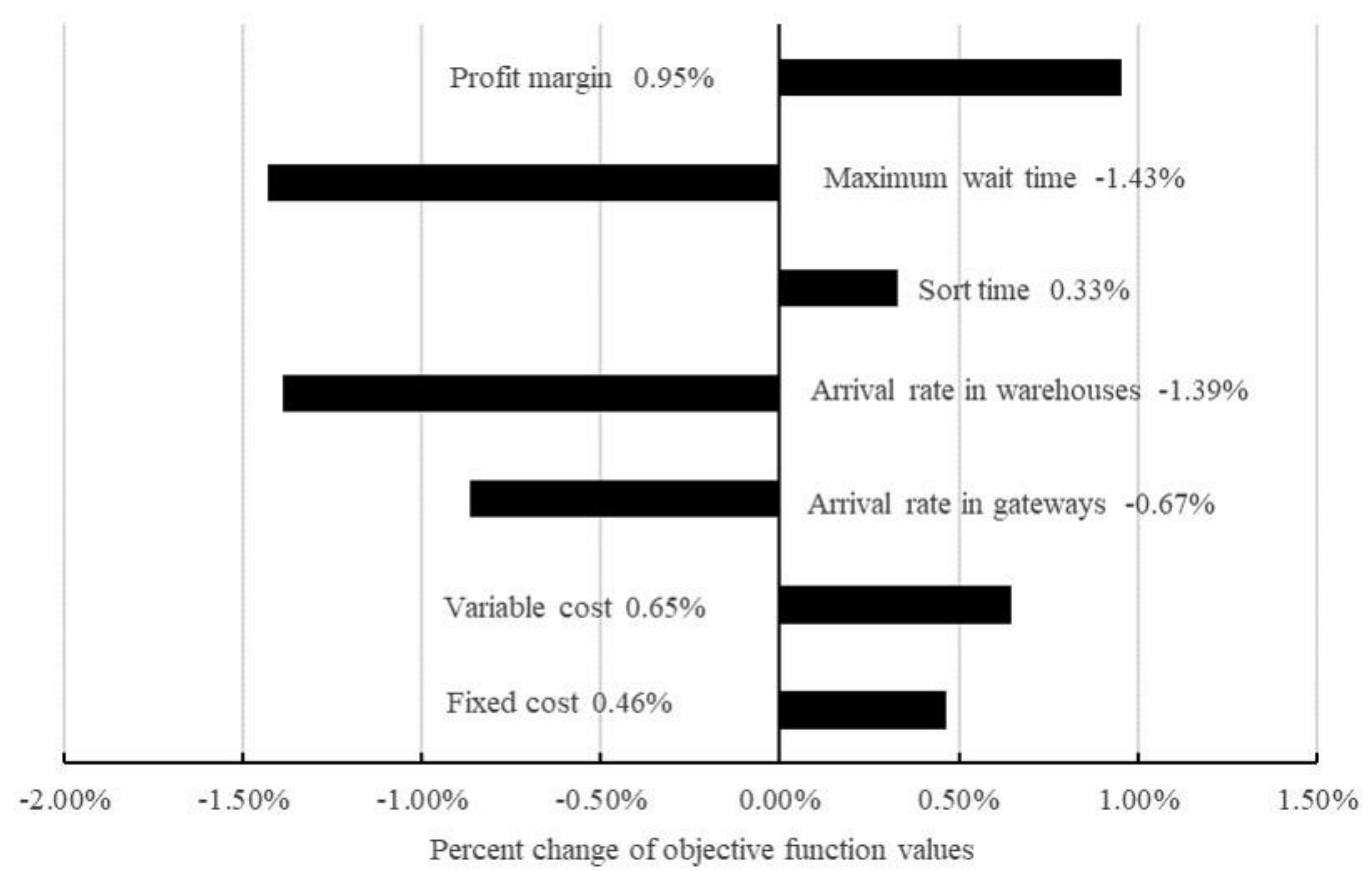

Figure 7. Effects of increasing parameters by one percent on the Objective 1 value of average truck distance per container. 


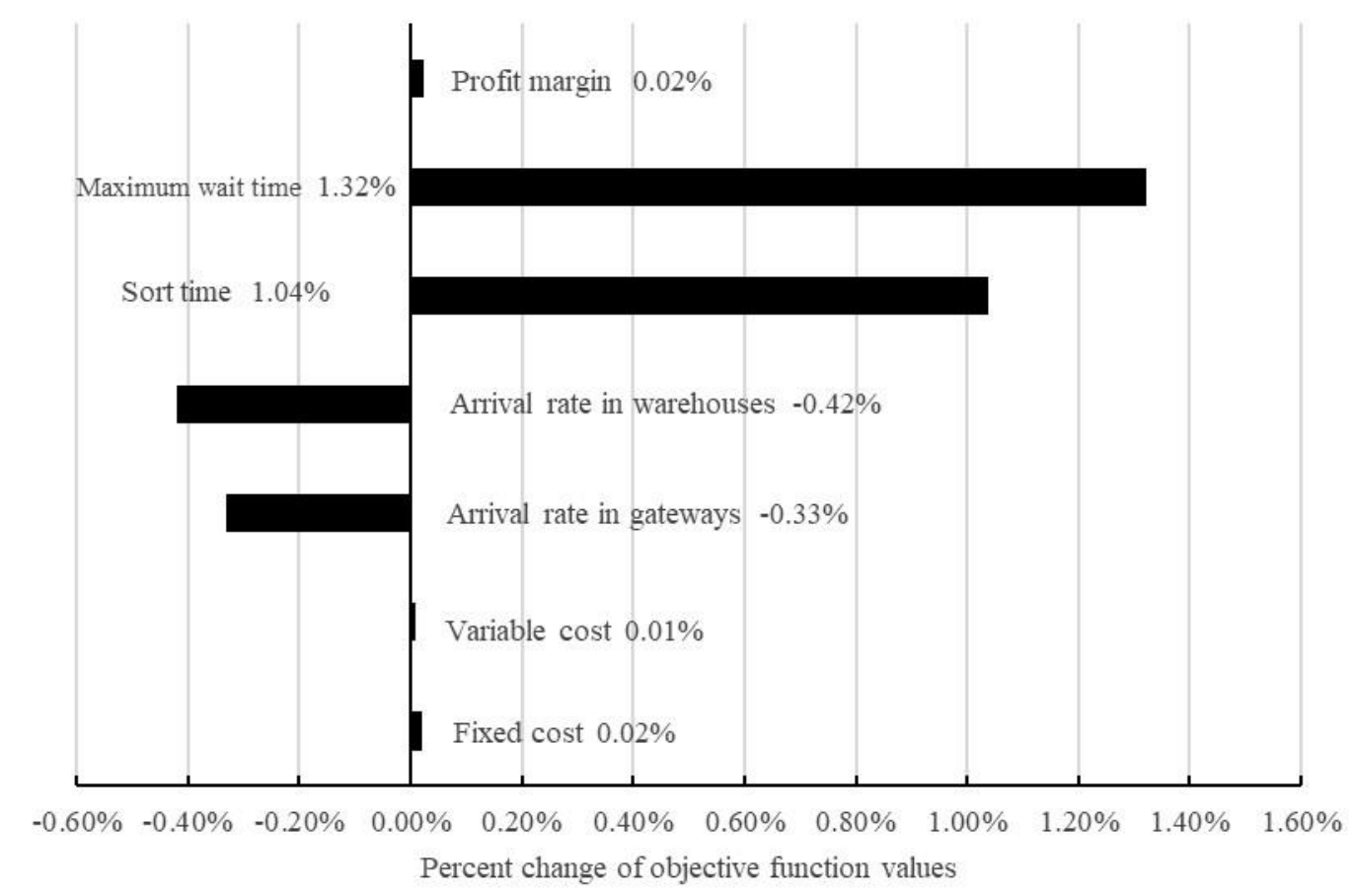

Figure 8. Effects of increasing parameters by one percent on the Objective 2 value of average truck lead time per container.

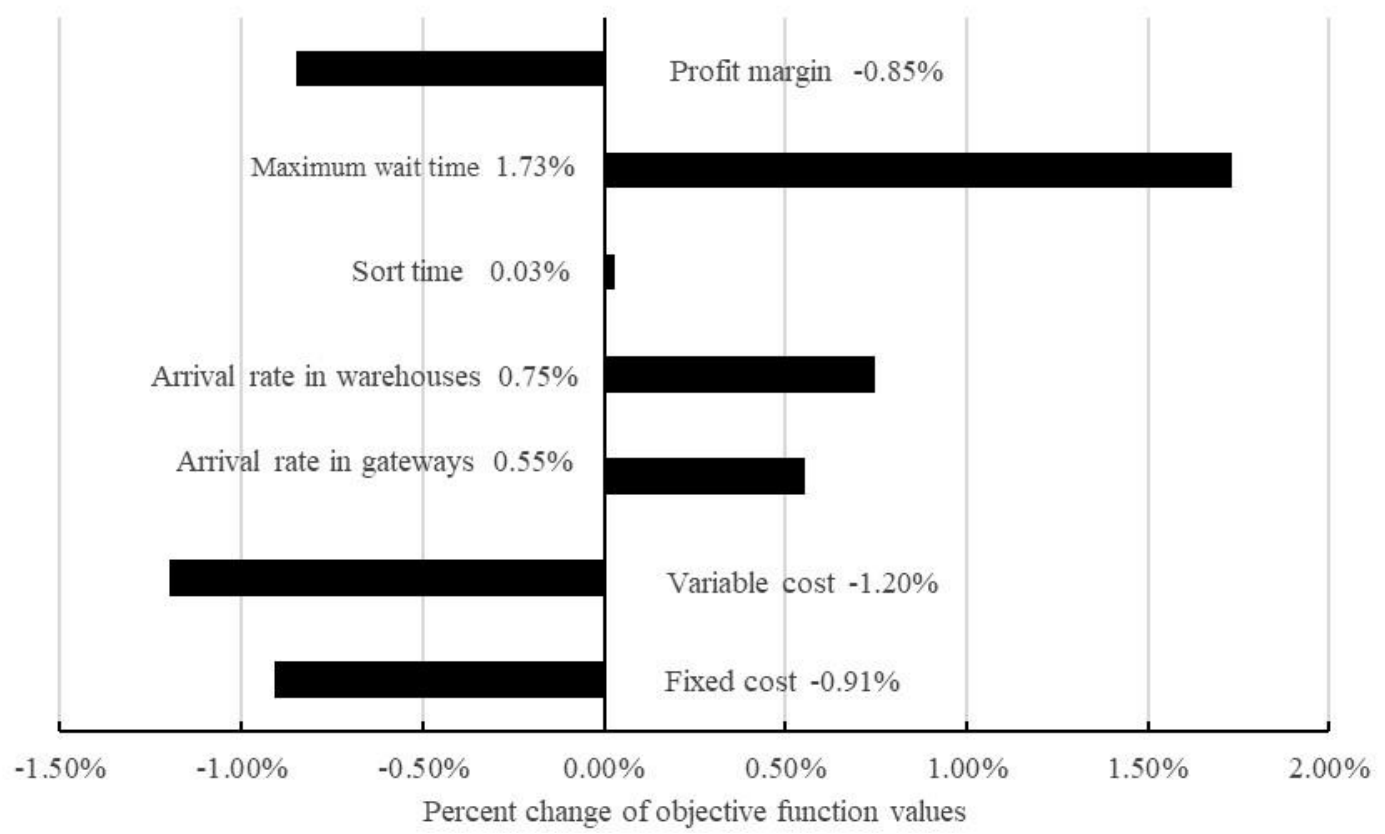

Figure 9. Effects of increasing parameters by one percent on the Objective 3 value of average truckload utilization. 


\subsection{Multi-objective optimization}

Next, we design a set of experiments to explore the trade space that provides relative valuations of multiple objectives concurrently, and we take a weighted sum approach to find Pareto efficiency. Three experiments are developed by focusing on any two of Objectives 1-3; that is, Objectives 4-6 as defined in Table 3. The weighting factors $\omega, \lambda$, and $\theta$ are varied from 0 to 1 with a step interval of 0.05 . Figure 10 plots a Pareto frontier for each of the bi-objective optimization scenarios along the trade-off lines between objectives.

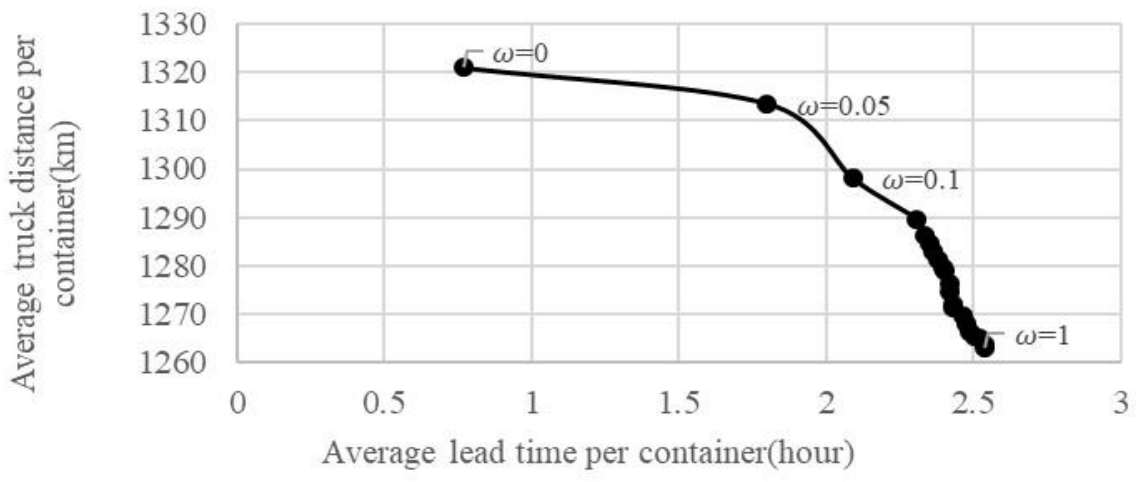

(a)

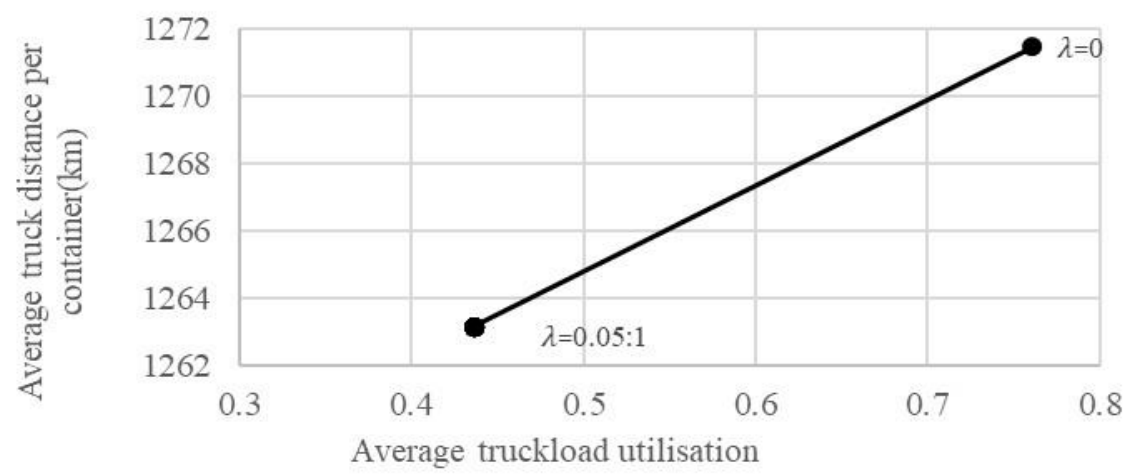

(b)

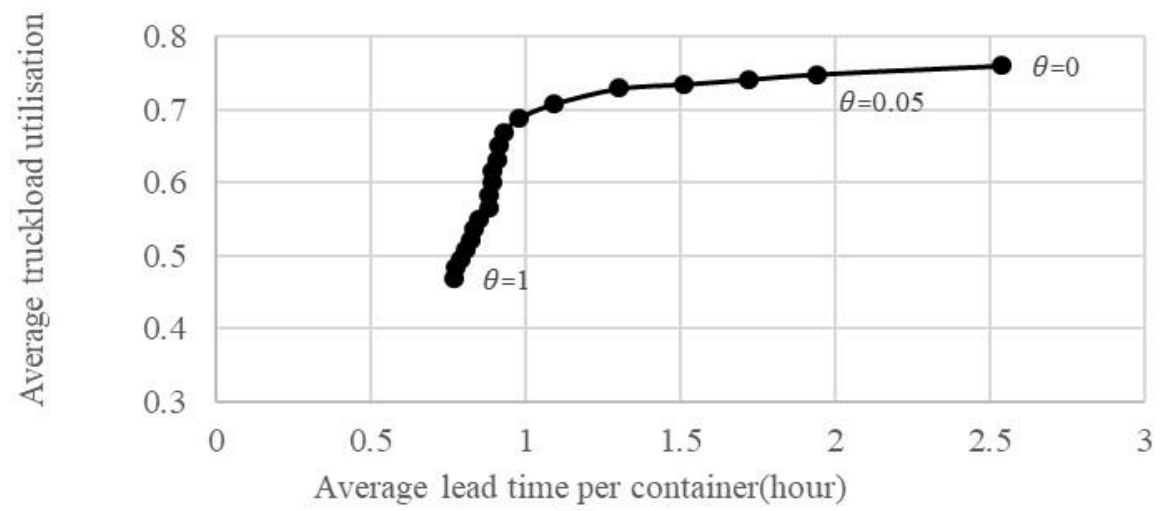

(c) 
Figure 10. Pareto front efficiencies in trade-spaces: (a) between truck distance per container and lead time per container (Objective 4); (b) between truck distance per container and truckload utilization (Objective 5); (c) between truckload utilization and lead time per container (Objective 6).

As $\omega, \lambda$, and $\theta$ increase from 0 to 1 , Figure 10(a) - which relates to Objective 4 - indicates the relationship between two competing Objectives 1 and 2 while there is a higher density of observations with more than two hours of average lead time per container. On the other hand, Figures 10(b) and 10(c) - which relate to Objectives 5 and 6 , respectively - show that higher truckload utilization accommodates an increase of truck distance or lead time per container, implicating the complementary relationships between each pair. There are still some unpopulated sections along the Pareto front in all three cases. As $\omega, \lambda$, and $\theta$ approach one, the points slowly converge to lower ends; particularly in Figure 10(b), 19 coincident points are on the left end. To achieve more suitably scaled relationships between the various objective criteria, multi-objective optimization is transformed into a single objective. More specifically, while keeping one single objective, the remaining measure(s) becomes constraints that are subject to a range of values obtained from the weighted sum approach in Figure 10.

Figure 11(a) illustrates the relevant results on the objective of average truck distance per container when limiting lead time per container in the range of $[0.8,2.8]$. In this case, the longer average lead time allows carriers to consolidate more shipments and therefore facilitates achieving the less average truck distance per container in the system. Figure 11(b) shows the higher utilization of truckload, enabling shorter average truck distance per container with a minimum value reached at 0.7 truckload utilization where $D$ (truck capacity, truck numbers $)=[9,44]$. Beyond this point, however, higher truckload utilization (0.78) incurs a farther truck distance with the optimal solution of less resources: $D$ (truck capacity, truck numbers) $=[8,35]$. It can be seen from Figure 11(c) that a higher truckload utilization indicates a longer average lead time per container. A truck can load more containers by raising the maximum limit of its fill rate which, in turn, entails potentially accruing additional times from the point of individual containers. 


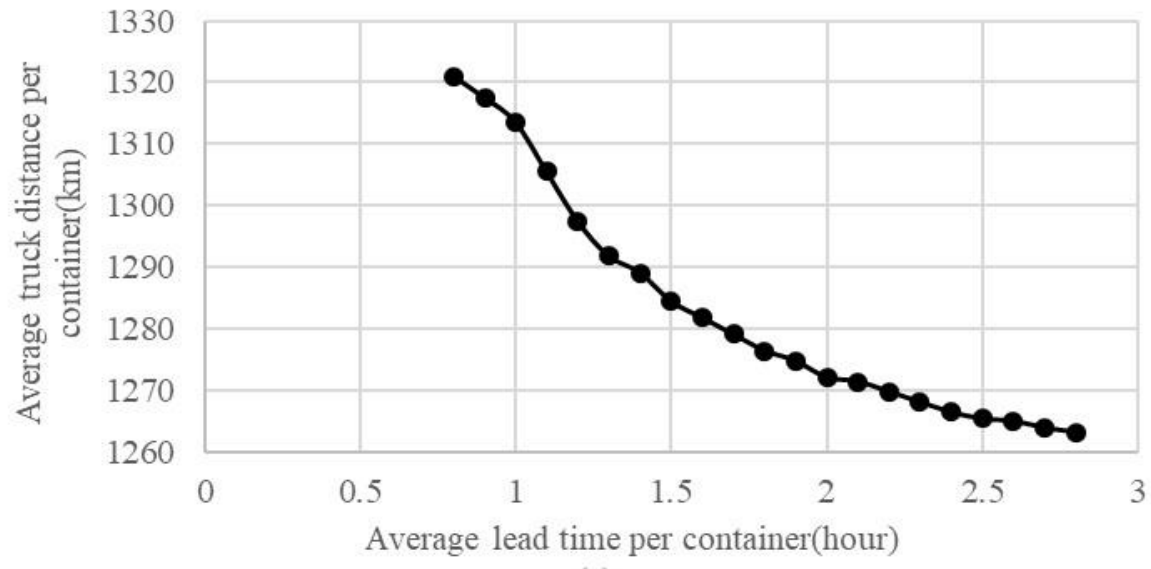

(a)

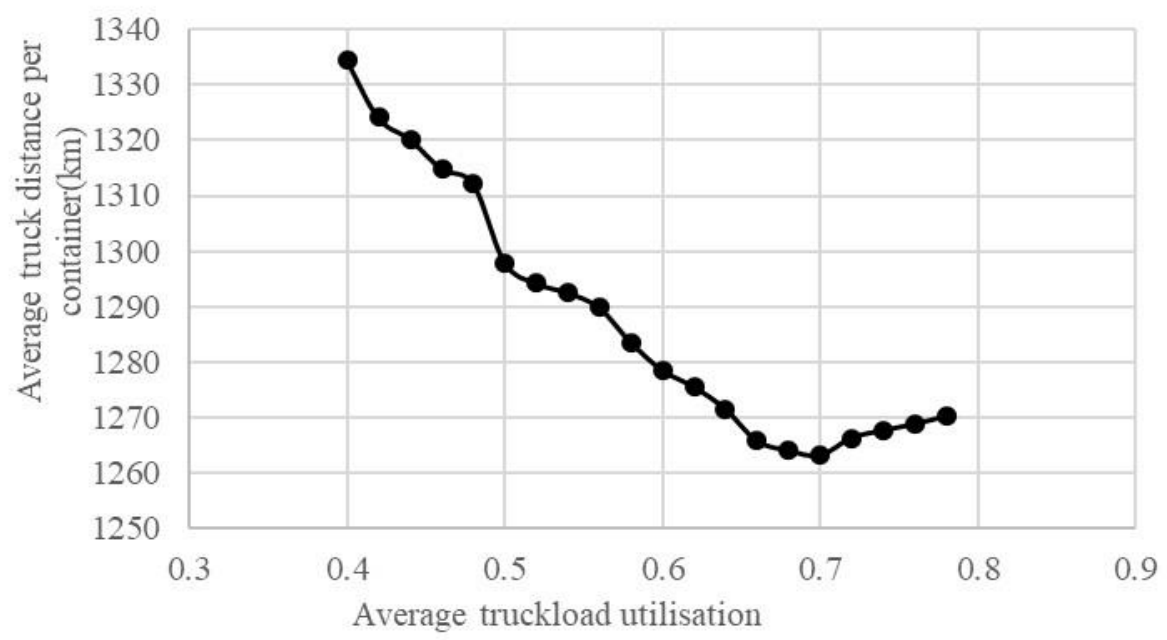

(b)

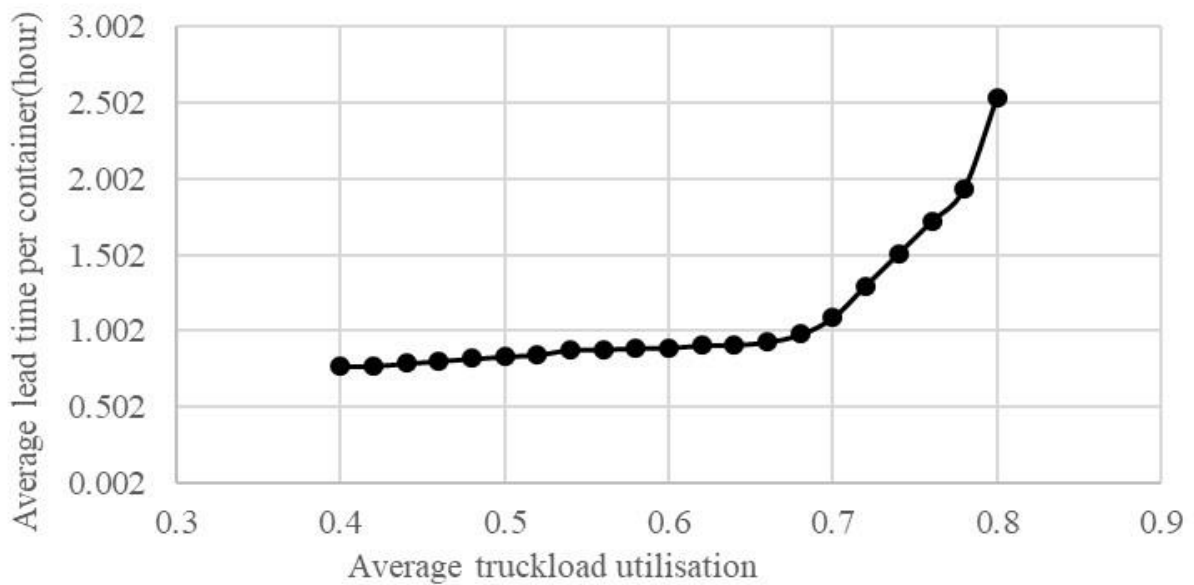

(c)

Figure 11. (a) truck distance per container subject to lead time per container; (b) truck distance per container subject to truckload utilization; and (c) lead time per container subject to truckload utilization. 
Finally, the objective value of average truck distance per container decreases as the constraints of average lead time and truckload utilization are relaxed. As shown in Figure 12, the lower average truck distance in the system is attributed to a longer average lead time and higher truckload utilization. We also note that the problem becomes infeasible when the average lead time per container is less than 0.9 hours and the average truckload utilization is greater than 0.66 . This implies that the optimal solution cannot be obtained at both extreme ends of the two objectives simultaneously.

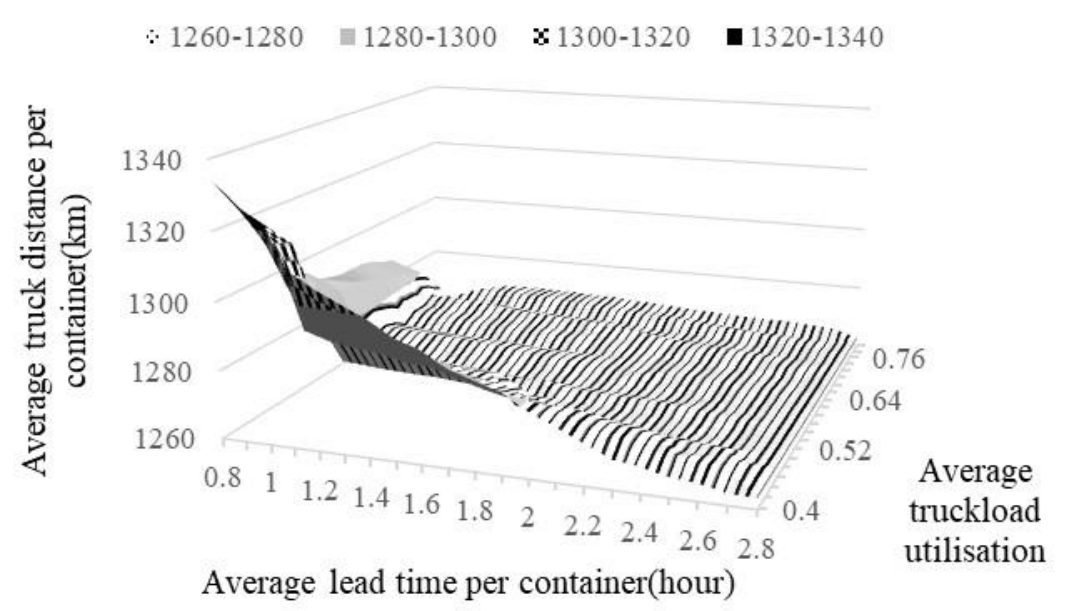

Figure 12. Average truck distance per container under the values of average lead time per container and average truckload utilization (Objective 7; Table 3).

\section{Discussions}

For freight transport planning, a PI-enabled logistics system has the potential to allocate and reallocate logistical resources such as containers, trucks and hubs in efficient and are environmentally sustainable ways. It achieves this through shared, open, and collaborative networks. Given that capacity sharing is a fundamental concept and core driver of the proposed PI modeling framework, increasing utilization and efficient load capacity consolidation becomes a focal point of our simulation study. In this paper, to address the prospect of PI, we develop a hybrid model that combines simulation with techniques used in the wider OR literature, namely, auction mechanisms and optimization. The hybrid model consists of a multi-agent simulation embedded with an optimization routine to represent the complex interplay involving uncertainty in a collaborative freight transportation system. Our model adopts an auction mechanism designed for assigning requests more efficiently to consolidate transports. To measure the effects of parameter settings in the system, a set of sensitivity analyses are conducted on 
three single objectives, i.e., average truck distance per container, average truck lead time per container, and average truckload utilization.

Some of the benefits of PI include lower truck driving distances and, consequently, faster transportation of loads from origins to destinations. From an environmental standpoint, a decrease in driving distance also contributes to a reduction in $\mathrm{CO} 2$ emissions. Nevertheless, under the PI scheme, it is observed that the number of container exchanges at hubs increases due to the higher instances of loading and unloading as indicated in Figures 7-9 with respect to the maximum wait time limit. We also note that the success of a PI-enabled logistic system is highly dependent on carrier profit margins, which would dictate their participation in the sharing scheme. Moreover, four multi-objectives have been explored by selectively combining them. When considering the joint effects of multiple objectives, some of the insights gleaned from our computational results are:

- There are trade-offs in the optimal solutions of decision variables including truck number and truck capacity, with regard to the objectives of average truck distance per container and average truckload utilization. The corresponding results indicate that more trucks and larger capacities contribute to a shorter average truck lead time per container.

- Parameters such as maximum wait time and arrival rate have positive impacts on enhancing the collaboration. The results from the sensitive analysis support these findings (in relation to Objectives 1-3). On the contrary, cost factors including profit margin, variable cost, and fixed cost adversely affect minimizing objective values.

- In multi-objective scenarios, relationships under multiple criteria assessment in collaborating shipments can be inferred as in Section 5.2. A longer average lead time allows carriers to achieve the less average truck distance per container. Increasing utilization of truckload gives the added benefit of shortening the average truck distance per container. Nevertheless, there is a trade-off between truckload utilization and lead time per container.

Fazili et al. reported driving times of routes required to transport containers from origin to destination with or without PI consideration, and the scenario with PI indicates an advantage of the average driving time being reduced by about $5.6 \%$. $^{22}$ This is comparable to our result presented in Table 6, which shows an average decrease in driving 
time of 9.7\%. Also, when PI is implemented in their model, Sarraj et al. claimed that the transportation fill rate gains up to $17 \%$ from a current level of $59 \%$ utilization of the load capacity of conventional transportation means. ${ }^{14}$ On a par with these results, the average truck utilization presented in Table 6 shows an average improvement of $15.3 \%$ across all six scenarios when a collaborative agreement of PI is in place.

Our findings also support recent practices that have evolved in the logistics industry for sharing space for freight transport in general. Multi-customer warehouses enable logistics providers to take advantage of economies of scale. For example, DHL operates warehouse space sharing in a single warehouse site where the logistics provider can use fewer resources to meet the needs of multiple customers. With integrated supply chain management, they developed a platform called DHL Spaces where e-commerce companies can operate a more flexible omnichannel warehousing strategy. ${ }^{44}$ On the forefront of transport capacity sharing, the digital freight companies such as Freightos, Convoy, and Loadsmart are brokering between shippers and carriers by matching loads with available capacity. Following a sharing economy approach, they provide the market access and transaction processing for both shippers and carriers, and facilitate effective sharing by embracing the openness and scale of transport capacity.

The limitations of this research are now discussed. First, the formulation of WDP in the auction mechanism considers only factors related to cost and truck fill rate. In future, our hybrid model can include other criteria in the truck selection stage. Second, the multi-objective optimization model (Section 4.2) used a simple weighted sum approach to determine the efficient Pareto frontiers. However, there are other methods such as Goal Programming (GP) ${ }^{45}$ and Multiobjective Evolutionary Algorithm based on Decomposition (MOEA/D), ${ }^{46}$ which could be explored and compared in future studies.

Future work could also extend the hybrid model to include the evaluation of logistics and supply-chain performance using Colored Petri Net based simulation approach. ${ }^{47}$ In addition, determining the weights of various objectives could reflect qualitative criteria in conjunction with a further understanding of stakeholders. For instance, Sencer and Karaismailoglu used analytical hierarchy process (AHP) to evaluate the criteria weights, both quantitative and qualitative, and rank alternatives resulting from the output of their simulation model. ${ }^{48}$ This is a separate procedure of the solution approach whereas an optimization routine is embedded on our simulation model in an integrated fashion. It would be worthwhile to compare the computational efficiency as well as the solution quality between the two methodologies. In another recent study, 
Sadeghi et al. ${ }^{49}$ developed a simulation model to discover bottlenecks in a port's rail container transport process and find beneficial configurations for use of rail for fast and large handling. Due to the fact that trains can bring considerably more goods than trucks, delivering cargo via the railway system would increase ports' handling capacities. As a result of the study, it was discovered that the port of interest could increase the share of rail up to $8 \%$ without spending on equipment or infrastructure, although optimization was not employed. A more general overview of the use of simulation and optimization for transportation systems is provided in De La Torre et al. ${ }^{50}$

While the logistics industry will continue to remain disruptive with innovations and technology development in the foreseeable future, its stakeholders have an essential role in paving the way for the sharing economy. In our day-to-day life, we observe the abundance of idle resources and sharing instead of owning becoming a growing trend, and logistics can serve as one of the main drivers of this advancement.

\section{Reference}

1. US Department of Commerce. US census bureau news - quarterly retail e-commerce sales, 3rd quarter. economic indicators division. CB19-170.

https://www.census.gov/retail/mrts/www/data/pdf/ec_current.pdf (2019, accessed 10 February 2020).

2. Anderson WP and Leinbach TR. E-commerce, logistics and the future of globalized freight. In: Leinbach TR and Capineri C (eds) Globalized freight transport: intermodality, $e$ commerce, logistics and sustainability, 2007; pp.167-187.

3. Beliën J, Boute R, Creemers S, et al. Collaborative shipping: logistics in the sharing economy. ORMS Today, 2017, p.20.

4. Chapman RL, Soosay C and Kandampully J. Innovation in logistic services and the new business model: a conceptual framework. Manag Serv Qual 2002; 12: 358-371.

5. Srinivasan A and Leveque P. Digitising freight - one truck at a time: A Frost \& Sullivan perspective on the emerging digital freight brokerage systems, https://ww2.frost.com/frostperspectives/digitising-freight-one-truck-time-frost-sullivan-perspective-emerging-digitalfreight-brokerage-systems (2016, accessed 20 June 2019)

6. Benjelloun A, Crainic TG and Bigras, Y. Towards a taxonomy of city logistics projects. Procd Soc Behv 2010; 2: 6217-6228.

7. Dablanc L. Goods transport in large European cities: Difficult to organize, difficult to modernize. Transp Res A-Pol 2007; 41: 280-285. 
8. Montreuil B, Meller RD and Ballot E. Physical internet foundations. In: Borangiu T, Thomas A and Trentesaux D. (eds) Service orientation in holonic and multi agent manufacturing and robotics. Berlin, Heidelberg: Springer, 2013, pp.151-166.

9. Ferrell W, Ellis K, Kaminsky P, et al. Horizontal collaboration: opportunities for improved logistics planning. Int $J$ Prod Res ahead of print 11 August 2019; DOI: 10.1080/00207543.2019.1651457.

10. Tolk A, Harper A, and Mustafee N. Hybrid models as transdisciplinary research enablers. Eur J Oper Res 2021; 291(3): 1075-1090.

11. Pan S, Trentesaux D, Ballot E, et al. Horizontal collaborative transport: survey of solutions and practical implementation issues. Int J Prod Res 2019; 57: 5340-5361.

12. Montreuil B. Toward a physical internet: Meeting the global logistics sustainability grand challenge. Logist Res 2011; 3: 71-87.

13. Rajahonka M, Bask A, Yawar SA, et al. The physical internet as enabler of new business models enhancing greener transports and the circular economy. In: Eftestøl-Wilhelmsson E, Sankari S and Bask A (eds) Sustainable and efficient transport. UK: Edward Elgar Publishing, 2019, pp.70-94.

14. Sarraj R., Ballot E, Pan S, et al. Interconnected logistic networks and protocols: Simulationbased efficiency assessment. Int J Prod Res 2014; 52: 3185-3208.

15. Hakimi D, Montreuil B and Labarthe O. Supply web: Concept and technology. In: Proceedings of 7th annual international symposium on supply chain management, Toronto, Canada, 2009, pp.1-21.

16. Oktaei P. Physical internet enabled transit centers: Business modeling and profitability assessment. Master Thesis, University of Laval, Québec, Canada, 2015.

17. Douma A, Schuur PC and Van Der Heijden MC. Applying revenue management to agentbased transportation planning. Report (BETA: Vol. 169), Universiteit Twente, Netherland, 2006.

18. Hakimi D, Montreuil B, Sarraj R, et al. Simulating a physical internet enabled mobility web: The case of mass distribution in France. In: Proceedings of 9th international conference on modeling optimization \& simulation, Bordeaux, France, 2012, pp.1-10.

19. Meller RD, Lin $\mathrm{YH}$ and Ellis KP. The impact of standardized metric physical internet containers on the shipping volume of manufacturers. IFAC P Ser 2012; 45: 364-371.

20. Cruijssen F, Cools M, and Dullaert W. Horizontal cooperation in logistics: opportunities and impediments. Transport Res E-Log 2007; 43: 129-142.

21. Krajewska MA, Kopfer H, Laporte G, et al. horizontal cooperation among freight carriers: request allocation and profit sharing. J Oper Res Soc 2008; 59: 1483-1491. 
22. Fazili M, Venkatadri U, Cyrus P, et al. Physical internet, conventional, and hybrid logistics systems: a routing optimisation-based comparison using the eastern Canada road network case study. Int J Prod Res 2017; 55: 2703-2730.

23. Gansterer M and Hartl R. Collaborative vehicle planning: a survey. Eur J Oper Res 2018; 268: $1-12$.

24. Mustafee $\mathrm{N}$ and Powell JH. From hybrid simulation to hybrid systems modelling. In: Proceedings of the Winter Simulation Conference, Gothenburg, Sweden, 9-12 December 2018, pp.1430-1439. IEEE.

25. Qiao B, Pan S and Ballot E. Dynamic pricing model for less-than-truckload carriers in the physical internet. J Intell Manuf 2019; 30: 2631-2643.

26. Pan S, Xu X and Ballot E. Auction based transport services allocation in physical internet: a simulation framework. In: Proceedings of 1st international physical internet conference, Québec, Canada, 2014, pp.1-13.

27. Wang $X$ and Kopfer H. Collaborative transportation planning of less-than-truckload freight: a route-based request exchange mechanism. OR Spectrum 2014; 36: 357-380.

28. Xu X, Pan S and Ballot E. Game theoretic contribution to horizontal cooperation in logistics. In: Proceedings of 4th international conference on information systems, logistics and supply chain, Québec, Canada 2012, pp.1-10.

29. Berger S and Bierwirth C. Solutions to the request reassignment problem in collaborative carrier networks. Transport Res E-Log 2010; 46: 627-638.

30. Chang Y and Makatsoris H. Supply chain modelling using simulation. Int J Simul Model 2001; 2: 24-30.

31. Axlerod R. Advancing the art of simulation in the social sciences. In: Conte R, Hegselmann R and Terna P (eds) Simulating Social Phenomena. Berlin, Germany: Springer-Verlag, 1997, pp. 21-40.

32. Shah N. Process industry supply chains: advances and challenges. Comput Chem Eng 2005; 29: 12251235 .

33. Macal CM and North MJ. Tutorial on agent-based modelling and simulation. J Simul 2010; 4: $151-162$.

34. Carson Y and Maria A. Simulation optimization: methods and applications. In: Proceedings of the 1997 winter simulation conference, 1997, pp. 118-126. Piscataway, NJ: IEEE.

35. April J, Glover F and Kelly J. Portfolio optimization for capital investment projects. In: Proceedings of the 2002 winter simulation conference, 2002, pp. 1546-1554. Piscataway, NJ: IEEE.

36. Better M, Glover F, Kochenberger G, et al. Simulation optimization: applications in risk management. Int J Inf Tech Decis 2008; 7: 571-587. 
37. Banks J, Carson JS, Nelson BL, et al. Discrete event systems simulation. 3rd ed. Englewood Cliffs, NJ: Prentice Hall, 2000.

38. Swisher JR, Jacobson SH, Hyden PD et al. A survey of simulation optimization techniques and procedures. In: Proceedings of the 2000 Winter Simulation Conference, 2000, pp. 119128. Piscataway, NJ: IEEE.

39. Brailsford S, Eldabi T, Kunc M, et al. Hybrid simulation modelling in operational research: a state-of-the-art review. Eur J Oper Res 2019; 278: 721-737.

40. Powell JH and Mustafee N. Widening requirements capture with soft methods: an investigation of hybrid M\&S studies in healthcare. J Oper Res Soc 2017; 68: 1211-1222.

41. Davidsson P, Henesey L, Ramstedt L, et al. An analysis of agent-based approaches to transport logistics. Transport Res C 2005; 13: 255-271.

42. Serrano-Hernandez A, Faulin J, Hirsch P, and et al. Agent-based simulation for horizontal cooperation in logistics and transportation: from the individual to the grand coalition. Simul Model Pract Th 2018; 85: 47-59.

43. Mustafee $\mathrm{N}$ and Bischoff EE. Analysing trade-offs in container loading: combining load plan construction heuristics with agent-based simulation. Int Trans Oper Res 2013; 20: 471-491.

44. Gesing B. Sharing economy logistics Technical Report. DHL Customer Solutions \& Innovation, Troisdorf, Germany. 2017.

45. Barichard V, Ehrgott M, Gandibleux X, et al. Multiobjective programming and goal programming: theoretical results and practical applications. Berlin, Germany: Spring Verlag, 2009.

46. De Farias LRC, Braga PHM, Bassani HF, et al. MOEA/D with uniformly randomly adaptive weights. In: Proceedings of the genetic and evolutionary computation conference, 2018, pp. 641-648. New York: ACM.

47. Drakaki M and Tzionas P. A Colored Petri Net-based modeling method for supply chain inventory management. Simulation, 2021. DOI:10.1177/00375497211038755.

48. Sencer A and Karaismailoglu A. A simulation and analytic hierarchy process based decision support system for air cargo warehouse capacity design. Simulation, 2021. DOI:10.1177/00375497211029915.

49. Sadeghi M, Bagheri M and Pishvaee MS. Evaluation of rail terminals in container ports using simulation: A case study. Simulation, 2021. DOI:10.1177/00375497211024731.

50. De La Torre R, Corlu C, Faulin J, Onggo B, and Juan A. Simulation, optimization, and machine learning in sustainable transportation systems: models and applications. Sustainability 2021; 13(3): 1551. 